Documentation et bibliothèques

DOCUMENTATION BIBLIOTHËQUES

\title{
Auteurs des comptes rendus, auteurs et titres des documents recensés
}

Numéro hors-série, 1993

URI : https://id.erudit.org/iderudit/1028592ar

DOI : https://doi.org/10.7202/1028592ar

Aller au sommaire du numéro

Éditeur(s)

Association pour l'avancement des sciences et des techniques de la documentation (ASTED)

\section{ISSN}

0315-2340 (imprimé)

2291-8949 (numérique)

Découvrir la revue

Citer ce document

(1993). Auteurs des comptes rendus, auteurs et titres des documents recensés. Documentation et bibliothèques, 37-71. https://doi.org/10.7202/1028592ar

Tous droits réservés ( $)$ Association pour l'avancement des sciences et des techniques de la documentation (ASTED), 1993
Ce document est protégé par la loi sur le droit d'auteur. L'utilisation des services d'Érudit (y compris la reproduction) est assujettie à sa politique d'utilisation que vous pouvez consulter en ligne.

https://apropos.erudit.org/fr/usagers/politique-dutilisation/ 


\section{Auteurs des comptes rendus*, auteurs et titres des documents recensés}

1300 documents audiovisuels sur les personnes handicapées

voir Office des personnes handicapées du Québec

\section{A}

A l'écoute de l'oeil : les collections iconographiques et les bibliothèques. (JeanRémi Brault)

$$
35 \text { (1989), no 4, p. 193-194 }
$$

(Abid, Abdelaziz). Lajeunesse, Marcel. Répertoire des écoles des sciences de l'information

26 (1980), no 4, p. 225-227

Abrégé de la classification décimale de Dewey. Nouvelle édition entièrement refondue et complétée à partir de la première version intégrale française et de la XIXe édition intégrale en langue anglaise voir Béthery, Annie

Abridged Dewey Decimal Classification and Relative Index

voir Dewey, Melvil

L'accès aux publications au Canada

voir Colloque sur l'accès aux publications au Canada, Québec, 1986

Accès aux publications gouvernementales québécoises et canadiennes : index permuté

voir Day, Mario

Accessibilité et utilisation des publications officielles dans les bibliothèques

voir Cherns, Jack J.

Actes du Colloque organisé par le comité des relations internationales de l'ASTED... Montréal, 16-17 novembre 1981

voir Information et documentation : attentes des pays en voie de développement. Information and documentation. the..

The African Book World \& Press : A Directory. Répertoire du livre et de la presse en Afrique. 2nd ed., rev. and greatly enl. (W. Dale Ward)
African Books in Print. Livres africains disponibles

voir Zell, Hans M.

(Alain, Jean-Marc). Québec. Ministère d'État au Développement culturel. Pour une politique québécoise de la recherche scientifique

26 (1980), no 2, p. 113-116

Alain, Jean-Marc. Pour une gestion adaptée des services documentaires. (Jean-Rémi Brault)

36 (1990), no 1, p. $37-39$

Alain, Jean-Marc. Une bibliothèque pour Montréal; rapport préliminaire sur la possibilité d'établir une . bibliothèque publique à la Place Desjardins, sous la direction de André Castonguay, par JeanMarc Alain (et autres). (Jacques Panneton)

20 (1974), no 1, p. 42-43

(Alain, Marc). Bonville, Jean de. Introduction aux ouvrages généraux de référence sur la communication et les mass media

22 (1976), no 1, p. $49-50$

Alexandre Vattemare trait d'union entre deux mondes

voir Revai, Elisabeth

Alix, Yves. Ouvrages de référence sur la musique et les phonogrammes. Guide à l'usage des bibliothécaires et discothécaires. (Micheline Perreault)

29 (1983), no 4, p. 169

(Allaire, Daniel). Brière, Jean-Marie. L'information gouvernementale au Québec : bibliographie analytique

25 (1979), no 1 , p. $48-49$

(Allaire, Daniel). Information et liberté. Rapport de la Commission d'étude sur l'accès du citoyen à l'information gouvernementale et sur la protection des renseignements personnels

$$
27 \text { (1981), no 4, p. 159-161 }
$$

Allard, Yvon. Écrits sur l'avenir. (Robert Chamberot)

29 (1983), no 1, p. $45-46$
Allard, Yvon. Paralittérature 1. (JaninaKlara Szpakowska)

22 (1976), no 1, p. $45-47$

American Library Association. Règles de catalogage anglo-américaines (2e éd.) (Pierre Manseau)

26 (1980), no 4, p. $218-220$

Amoikon, Michel K. Structures, organisation et animation culturelle dans les bibliothèques en Côte d'Ivoire. (Jean de Chantal)

22 (1976), no 4, p. 199-203

Amrouni, Dominique. Dictionnaire anglaisfrançais de l'électronique et de l'électrotechnique, par Pierre Renyi et Dominique Amrouni. (Claude Fournier)

33 (1987), no 1, p. 29-30

Analyse des stéréotypes masculins et féminins dans les manuels scolaires au Québec

voir Dunnigan, Lise

Anatomie de la presse ethnique au Canada voir Lacroix, Jean-Michel

Animer la lecture : pour faire lire...; guide pratique voir Beauchesne, Yves

L'année politique au Québec, 1987/1988, sous la direction de Denis Monière, en collaboration avec le journal Le Devoir. (Gaston Bernier)

36 (1990), no 3, p. $107-108$

Les années '80. au service des clientèles. (Alain Perrier)

28 (1982), no 3, p. 133-135

Annotated Bibliography of Genealogical Works in Canada

voir Mennie-de-Varennes, Kathleen

Annuaire des bibliothèques canadiennes voir Canadian Library Yearbook = Annuaire des bibliothèques canadiennes

* Les auteurs de comptes rendus apparaissent toujours entre parentheses. 
Annuaire du cinéma québécois 1990

voir Jutras, Pierre sous la dir. de

Annuaire du Québec, 1979/1980. (Gaston Bernier)

$$
28 \text { (1982), no 4, p. } 172
$$

Antennes, la revue québécoise des communications. 1976- .(Jean de Bonville)

$$
22 \text { (1976), no 3, p. 145-146 }
$$

(Antonelli, Claudio). Vincke, Christian. Problèmes de droit d'auteur en éducation, par Christian Vincke. Avec la collaboration de Pierre A. Côté et Victor Nabhan

$$
25 \text { (1979), no 1, p. 49-50 }
$$

Arbido-B. (Jean-Rémi Brault)

$$
33 \text { (1987), no 1, p. } 31-32
$$

Arbido-R. (Jean-Rémi Brault)

33 (1987), no 1, p. 31-32

L'architecture française; recherche - formes intérieures - arts - urbanisme (...) nos 369370. (Fernand C. Magnan)

$$
20 \text { (1974), no 1, p. } 48-50
$$

Les archives au $X X e$ siècle. Une réponse aux besoins de l'administration et de la recherche

voir Couture, Carol

Archives des femmes du Québec; guide sommaire

voir Lamothe, Madeleine

Archives écrites d'origine privée conservées au Centre d'archives de Québec et de Chaudière-Appalaches

voir Québec. Archives nationales

Archives nationales

voir Québec. Archives nationales

Archives nationales du Canada. Division des manuscrits. Guide des archives littéraires. (Michel Biron)

$$
35 \text { (1989), no 4, p. 192-193 }
$$

Archives publiques

voir Canada. Archives publiques

Archives publiques du Canada. Guide des sources d'archives sur le Canada français au Canada. (Pierre Savard)
Archives publiques du Canada. Union List of Manuscripts in Canadian Repositories I Catalogue collectif des manuscrits des archives canadiennes. (Jacques Ducharme)

$$
22 \text { (1976), no 1, p. } 47-49
$$

Archivistique québécoise voir Beaudin, François

Arguin, Louise Gagnon voir Gagnon-Arguin, Louise

(Arnoldi, Monique). Etats-Unis. Library of Congress. Subject Cataloging Division. Introduction à la huitième édition de Library of Congress Subject Headings

$$
26 \text { (1980), no 4, p. } 220-221
$$

Assemblée nationale

voir Québec. Assemblée nationale

Assemblée nationale. Bibliothèque de la législature.

voir Québec. Bibliothèque de la législature

Association française des documentalistes et bibliothécaires spécialisés. Manuel du bibliothécaire documentaliste travaillant dans les pays en développement. (Bernard Descamps)

24 (1978), no 4, p. $209-210$

Association française des documentalistes et des bibliothécaires spécialisés. Édition électronique et documentation... du plomb à l'électron. (Gilles Deschâtelets)

$$
31 \text { (1985), no 4, p. 170-171 }
$$

Association internationale pour le développement de la documentation, des bibliothèques et des archives en Afrique. Conférence sur les problèmes de la planification et de l'organisation des archives, bibliothèques et centres de documentation en Afrique, Abidjan, 11-17 septembre 1972. (Jean de Chantal)

$$
22 \text { (1976), no 4, p. 199-203 }
$$

Association pour l'avancement des sciences et des techniques de la documentation

voir Asted

Asted. Commission des bibliothèques spécialisées et de recherche. Répertoire des outils documentaires dans les centres de documentations. (Nicole Côté)

27 (1981), no 3, p. 126-127
ASTED. Travaux du $10 \mathrm{e}$ congrès tenu à Montréal du 27 au 30 octobre 1983. Audelà de l'information : la communication. (Jean de Bonville)

32 (1986), no 3, p. 97-98

Au nom du livre; analyse sociale d'une profession : les bibliothécaires voir Seibel, Bernadette

Aubin, Paul. Bibliographie de l'histoire du Québec et du Canada 1966-1975. Avec la collaboration de Paul-André Linteau, de l'équipe de bibliographie de la Revue d'histoire de l'Amérique française et de la Société Microfor Inc. (Gaétan Drolet et Gilles Gallichan)

28 (1982), no 3, p. $128-130$

Audiovisuel, pédagogie et communication voir Bourron, Yves

(Auger, Maurice). Durance. Cynthia J. Vers l'établissement d'un réseau de communication bibliographique au Canada 29 (1983), no 2, p. 77-78

(Auger, Roland). Houde, Denis. Index rétrospectif, 1955-1979

30 (1984), no 1, p. 35

Awad, Amal. Les bibliothèques canadiennes à l'ère de l'automatisation : synthèse bibliographique 1970-72, par Colette Rivet-Panaccio, Amal Awad et Robert Cardinal. (Marcel Fontaine)

19 (1973), no 2, p. 93-94

B

B.I.T.

voir Bureau international du travail

(Bachand, Michelle). Kallmann, Helmut et al. Encyclopédie de la musique au Canada

30 (1984), no 2, p. 61-62

Barbeau, Victor. Dictionnaire bibliographique du Canada français, par Victor Barbeau et André Fortier. (Yvan Lamonde) 21 (1975), no 3, p. 172-173

Barut, Catherine. Bibliothèques dans la rue. Une expérience des bibliothèques municipales de la Ville de Genève, par Catherine Barut et Isabelle Wenger. (Christiane Charette)

33 (1987), no 2, p. 71-72 
"Bases de données et centres serveurs", Documentaliste: Sciences de l'information, v.17, no 6, (novembre-décembre 1980), p.183-222. (Christian Bielle)

$$
27 \text { (1981), no 3, p. 128-130 }
$$

Bâtir l'avenir. Les communications au Québec : recherche et développement. Bilan et perspectives. (Jean de Bonville)

$$
29 \text { (1983), no 2, p. 84-88 }
$$

Beauchesne, Yves. Animer la lecture : pour faire lire...; guide pratique. (René Gélinas)

33 (1987), no 1, p. 32

Beaudin, François (comp.). Archivistique québécoise. (Nicole Dufresne)

$$
22 \text { (1976), no 2, p. 98-99 }
$$

Beaudiquez, Marcelle. Inventaire général des bibliographies nationales rétrospectives $=$ Retrospective National Bibliographies : International Directory. (Jean-Rémi Brault)

$$
33 \text { (1987), no 3, p. 104-105 }
$$

Beaudiquez, Marcelle. Ouvrages de référence pour les bibliothèques publiques : répertoire bibliographique, par Marcelle Beaudiquez et Anne Zundel-Ben-Khemis. (Gaston Blais)

21 (1975), no 2, p. 108-109

(Beaulieu, André). Lacroix, Jean-Michel. Anatomie de la presse ethnique au Canada

36 (1990), no 1, p. $36-37$

Beaulieu, André. La presse québécoise des origines à nos jours, 1920-1934, 19351944, par André Beaulieu et Jean Hamelin. (Jacques Guay)

31 (1985), no 4, p. 172-173

Beaulieu, André. La presse québécoise des origines à nos jours. Tome premier : 17641859, par André Beaulieu et Jean Hamelin. (Marcel Lajeunesse)

19 (1973), no 3, p. 138

Beaumarchais, Jean-Pierre de. Dictionnaire des littératures de langue française, par Jean-Pierre de Beaumarchais, Daniel Couty et Alain Rey. (Pierre Giguère)

31 (1985), no 2, p. 74-75

(Beauregard, Louise). Reboul, Jacquette. Du bon usage des bibliographies

20 (1974), no 4, p. 207-208
Beilke, Patricia F. Directives pour la conception et l'organisation de médiathèques scolaires, par Frances Laverne Carroll et Patricia F. Beilke. (Paulette Bernhard)

29 (1983), no 1, p. $43-44$

Bélanger, Marcel. L'utilisation des moyens didactiques imprimés et audiovisuels par les professeurs au secondaire : une étude préliminaire, par Marcel Bélanger et HoaThuy Pham-Dang. (Georgette Lapointe et Rosaire Pelletier)

23 (1977), no 3, p. 171-173

Bélanger, Nycole. "La documentation", Méta (numéro spécial), v.25, no 1, (mars 1980), sous la direction de Nycole Bélanger. (Marc Morin et Denis Rousseau) 27 (1981), no 1, p. 37-39

Bélanger, Rita. Règles de catalogage anglo-américaines (...) sous la direction de Paule Rolland-Thomas avec la collaboration de Pierre Deslauriers. Traduction de Rita Bélanger. (Yvon Richer) 19 (1973), no 4, p. 177-178

(Bélisle, Alvine). Soriano, Marc. Guide de la littérature pour la jeunesse

22 (1976), no 3, p. 150-153

Bélisle, Alvine. Guide de lecture pour les jeunes 5 à 13 ans. (Henriette Dostaler) 19 (1973), no 3, p. 136-137

Bélisle, Louis-Alexandre. Dictionnaire nordaméricain de la langue française. (Noëlle Guilloton)

$$
28 \text { (1982), no 3, p. } 135-136
$$

(Bélisle-Laflèche, Michelle). St-Amour, Jean-Pierre F. L'Outaouais québécois. Guide de recherche et bibliographie sélective

$$
25 \text { (1979), no 1, p. 51-53 }
$$

Bellemare, Louis. L'information électronique au Québec : guide pratique des services d'information en ligne, par Louis Bellemare et Léo Filion. (Yves Tessier) 33 (1987), no 4, p. 137-138

Bellen, Liana Van der voir Van der Bellen, Liana

Ben-Gera, Michel. La fréquentation des bibliothèques et des librairies au Canada, par Michel Ben-Gera, Peat Warwick and Partners et Brian L. Kingsley. (Gilbert Gagnon)

$$
27 \text { (1981), no 4, p. 164-166 }
$$

Ben-Khemis, Anne Zunder

voir Zunder-Ben-Khemis, Anne

Bergeron, Alain. Les habitudes de lecture des québécois. (Réal Messier)

$$
20 \text { (1974), no 1, p. 47-48 }
$$

Bergeron, Christian. Les centres de ressources éducatives dans l'enseignement secondaire : exploration de la diversité des modèles, des situations et des problèmes locaux, par Christian Bergeron et Hoa-Thuy Pham-Dang. (Georgette Lapointe et Rosaire Pelletier)

23 (1977), no 3, p. 171-173

(Bergeron, Louisette). Charbonneau, Hélène. Livres en langue française pour les jeunes

32 (1986), no 4, p. 158-160

(Bergeron-Choquette, Louisette). Durand, Marion. L'image dans le livre pour enfants, par Marion Durand et Gérard Bertrand

23 (1977), no 1, p. $48-51$

(Bergeron-Choquette, Louisette). L'enfant, l'image et le récit. Ouvrage collectif sous la direction de Denise Escarpit

25 (1979), no 3, p. 169-171

(Bergevin, Gilles). Thériault, Michel. Choix et acquisition des documents au Québec. Volume 1: Principes et applications

23 (1977), no 3, p. 169-170

Bernard Valiquette

voir Chantigny, Louis

(Bernhard, Paulette). Carroll, Frances Laverne. Directives pour la conception et l'organisation de médiathèques scolaires. par Frances Laverne Carroll et Patricia F. Beilke

29 (1983), no 1, p. $43-44$

(Bernhard, Paulette). Lévesque, Raymond. La bibliographie d'un plan de cours de niveau collégial et les paradigmes éducationnels pour une nouvelle définition et un nouveau cadre théorique de la bibliographie

34 (1988), no 3, p. 113-115

(Bernhard, Paulette). Une invitation : la bibliothèque scolaire mise à profit. Réalisation, Anne Bernier.

34 (1988), no 3, p. 109-110 
Bernier, Anne. Une invitation : la bibliothèque scolaire mise à profit. Réalisation, Anne Bernier. (Paulette Bernhard et Patrick Delobel)

34 (1988), no 3, p. $109-110$

(Bernier, Gaston). A Geo-documentary Profile of the Commonwealth Developing Countries

38 (1992), no 3, p. 173-174

(Bernier; Gaston). Annuaire du Québec, 1979/1980

$$
28 \text { (1982), no } 4 \text {, p. } 172
$$

(Bernier, Gaston). Bonin, Claude-André et al. Lexique d'information documentaire = Lexicon of Information and Documentation

32 (1986), no 4, p. 160-161

(Bernier, Gaston). Bourgault, Jacques. Guide de recherche documentaire en matière de publications parlementaires et gouvernementales du Québec

31 (1985), no 1, p. 40-41

(Bernier, Gaston). Bulletin signalétique d'information administrative

$$
30 \text { (1984), no 1, p. } 28-29
$$

(Bernier, Gaston). Canadian Library Yearbook = Annuaire des bibliothèques canadiennes

$$
31 \text { (1985), no 4, p. 167-168 }
$$

(Bernier, Gaston). Cherns, Jack J. Accessibilité et utilisation des publications officielles dans les bibliothèques

$$
32 \text { (1986), no 1-2, p. 60-61 }
$$

(Bernier, Gaston). Corbeil, Jean-Claude. Dictionnaire thématique visuel

$$
33 \text { (1987), no 3, p. 105-107 }
$$

(Bernier, Gaston). France. Commission de coordination de la documentation administrative. Dix ans d'activité; cinquième rapport au Premier ministre

$$
29 \text { (1983), no 3, p. } 119-120
$$

(Bernier, Gaston). Gagné, Raymond. Publications parlementaires québécoises : catalogue collectif

$$
32 \text { (1986), no 1-2, p. } 61
$$

(Bernier, Gaston). Index analytique du journal Le Monde diplomatique, 1954-1983 30 (1984), no 4, p. 141-142
(Bernier, Gaston). L'année politique au Québec, 1987/1988, sous la direction de Denis Monière, en collaboration avec le journal Le Devoir

36 (1990), no 3, p. 107-108

(Bernier, Gaston). La gestion des publications officielles

$$
37 \text { (1991), no 1, p. } 41
$$

(Bernier, Gaston). Laghzali, Mohamed. Guide de recherche documentaire en science politique, par Mohamed Laghzali et Evelyne Tardif

$$
34 \text { (1988), no 2, p. } 80-81
$$

(Bernier, Gaston). Liste mensuelle des publications du gouvernement du Québec

30 (1984), no 1, p. $28-29$

(Bernier, Gaston). Maillet, Lise. Provincial Royal Commissions and Commissions of Inquiry, 1867-1982 : a selective bibliography $=$ Commissions royales provinciales et commissions d'enquête, 1867-1982 : bibliographie sélective

$$
32 \text { (1986), no 3, p. 102-103 }
$$

(Bernier, Gaston). Profils géo-documentaires des États francophones du Sud. (Troisième édition rev. et augm.) 38 (1992), no 3, p. 173-174

(Bernier, Gaston). Veyron, Michel. Dictionnaire canadien des noms propres 37 (1991), no 4, p. 164-165

Bernier, Gérald. Le Québec en chiffres de 1850 à nos jours, par Gérald Bernier et Robert Boily. (Roger de la Sablonnière) 35 (1989), no 3, p. 121-123

Bernier, Gérald. Le Québec en transition : 1760-1867 : bibliographie thématique, par Gérald Bęrnier et Robert Boily. (Monique Mailloux)

$$
34 \text { (1988), no 4, p. 159-162 }
$$

Bernier, Silvie. Du texte à l'image : le livre illustré au Québec. (Jean-Rémi Brault)

$$
37 \text { (1991), no 3, p. } 125
$$

Bertrand, Gérard. L'image dans le livre pour enfants, par Marion Durand et Gérard Bertrand. (Louisette Bergeron- Choquette)

$$
23 \text { (1977), no 1, p. 48-51 }
$$

(Bertrand-Gastaldy, Suzanne). Chaumier, Jacques. Le traitement linguistique de l'information documentaire : l'analyse documentaire

24 (1978), no 3, p. 148-151
(Bertrand-Gastaldy, Suzanne). Guinchat, Claire. Introduction générale aux sciences et techniques de l'information et de la documentation, par Claire Guinchat et Michel Menou

29 (1983), no 1, p. $41-43$

(Bertrand-Gastaldy, Suzanne). Québec. Assemblée nationale. Journal des débats. Index 1988/1989

37 (1991), no 4, p. 167-168

Bérubé, Robert-Claude. Une sélection de 100 films tirés de romans en vidéocassettes. (Claude Fournier)

38 (1992), no 4, p. 215

Besoins en information et en services documentaires chez les jeunes de 14-19 ans

voir Szpakowska, Janina-Klara

Béthery, Annie. Abrégé de la classification décimale de Dewey. Nouvelle édition entièrement refondue et complétée à partir de la première version intégrale française et de la XIXe édition intégrale en langue anglaise. (Rosaire Pelletier)

29 (1983), no 4, p. 165

Bethery, Annie. Les périodiques, par Annie Bethery et Jacqueline Gascuel. Avec la collaboration de Michèle Ganot et al. (Huguette Desmarais)

25 (1979), no 3, p. 167-169

Bibliocom 1976; bibliographie internationale de la documentation en langue française sur la communication voir Bonville, Jean de

Bibliocom 1977; bibliographie internationale de la documentation en langue française sur la communication voir Bonville, Jean de

Bibliographie annotée d'ouvrages de référence en usage au bureau de la bibliographie rétrospective voir Mailloux, Pierre

Bibliographie annotée d'ouvrages généalogiques au Canada = Annotated Bibliography of Genealogical Works in Canada voir Mennie-de-Varennes, Kathleen

La bibliographie d'un plan de cours de niveau collégial et les paradigmes éducationnels pour une nouvelle définition et un nouveau cadre théorique de la bibliographie

voir Lévesque, Raymond 
Bibliographie de bibliographies québécoises

voir Bibliothèque nationale du Québec

Bibliographie de l'Abitibi-Témiscamingue

voir Gourd, Benoit-Beaudry

Bibliographie de l'histoire de l'éducation au Québec des origines à 1960

voir Houyoux, Philippe

Bibliographie de l'histoire du Québec et du Canada 1966-1975

voir Aubin. Paul

Bibliographie de la critique de la littérature québécoise et canadienne-française dans les revues canadiennes (1974-1978)

voir Dionne, René

\section{Bibliographie de la Gaspésie \\ voir Desjardins, Marc}

Bibliographie du Québec, 1821-1967. (Liana Van der Bellen)

$$
27 \text { (1981), no 1, p. 35-36 }
$$

Bibliographie québécoise sur les personnes handicapées

voir Janik, Sophie

Bibliographie sur les personnes âgées : répertoire des livres et des périodiques de la Bibliothèque de l'Université Laval voir Vinet, Bernard

Bibliothèque de France, bibliothèque ouverte. (Jean-Rémi Brault)

37 (1991), no 3, p. 121-122

Bibliothèque de la législature

voir Québec. Bibliothèque de la législature

La bibliothèque idéale, sous la direction de Pierre Boncenne. (Jean-Rémi Brault)

35 (1989), no 3, p. 125-126

Bibliothèque nationale du Canada. Groupe de travail sur le catalogue collectif canadien. Rapport final soumis au directeur général de la Bibliothèque nationale. (Alain Boucher)

24 (1978), no 1, p. 44-45

Bibliothèque nationale du Canada. Groupe de travail sur le catalogue collectif canadien. Sous-groupe de travail sur les catalogues collectifs de publications en série. Rapport. (Alain Boucher)

24 (1978), no 1, p. 44-45
Bibliothèque nationale du Canada. Groupe de travail sur le service de bibliothèque aux handicapés. Rapport. (Jean-Rémi Brault) 24 (1978), no 1, p. $41-43$

Bibliothèque nationale du Canada. Répertoire des bibliothèques canadiennes. 1Bibliothèques du gouvernement fédéral / Canadian Library Directory. 1- Federal Government Libraries. (Richard Paré)

20 (1974), no 4, p. 208-209

Bibliothèque nationale du Québec voir aussi Québec. Bibliothèque nationale

Bibliothèque nationale du Québec. Catalogue des manuscrits, dressé par le département des manuscrits. (Gisèle Hogue)

25 (1979), no 2, p. 105-107

Bibliothèque nationale du Québec. Radar, répertoire analytique d'articles de revues du Québec. (Lise Brunet)

19 (1973), no 2, p. 91-93

Bibliothèque nationale du Québec. Répertoire des périodiques québécois : 1re partie, par Ginette Henry. (Colette RivetPanaccio)

21 (1975), no 4, p. 227-228

La Bibliothèque nationale, mémoire de l'avenir

voir Blasselle, Bruno

La bibliothèque publique en milieu rural et dans les petites agglomérations voir Germanaud, Marie-Claire

La bibliothèque Saint-Sulpice, 1910-1931 voir Lassonde, Jean-René

La bibliothèque, miroir de l'âme, mémoire du monde

voir Figuier, Richard

Les bibliothèques canadiennes à l'ère de l'automatisation : synthèse bibliographique 1980-72

voir Rivet-Panaccio, Colette

Bibliothèques dans la rue. Une expérience des bibliothèques de la Ville de Genève voir Barut, Catherine

Les bibliothèques dans trois pays de l'ouest africain (Guinée, Mali, Sierra Leone)

voir Lalande-Isnard, F.

Les bibliothèques de collectivités à Montréal (17e-19e siècle) : sources et problèmes

voir Lamonde, Yvan
Les bibliothèques des collèges d'enseignement général et professionnel du Québec : étude de leur évolution, (19691983)

voir Lajeunesse, Marcel

Les bibliothèques personnelles au Québec : inventaire analytique et préliminaire des sources

voir Lamonde, Yvan

Les bibliothèques populaires voir Richter, Noë

Les bibliothèques publiques canadiennes de 1979 à 1983 : analyse comparative de certaines données statistiques

voir Mittermeyer, Diane

Les bibliothèques scolaires québécoises. Plus que jamais.

voir Québec. Ministère de l'Éducation. Direction générale de l'évaluation et des ressources didactiques

Les bibliothèques universitaires. Rapport au ministre d'État, ministre de l'Education nationale, de la Jeunesse et des Sports voir Miquel, André

(Bielle, Christian). "Bases de données et centres serveurs", Documentaliste : Sciences de l'information, v.17, no 6 , (novembre-décembre 1980), p. 183-222

27 (1981), no 3, p. 128-130

(Biron, Michel). Archives nationales du Canada. Division des manuscrits. Guide des archives littéraires 35 (1989), no 4, p. 192-193

(Blais, Gaston). Beaudiquez, Marcelle. Ouvrages de référence pour les bibliothèques publiques : répertoire bibliographique, par Marcelle Beaudiquez et Anne Zundel-Ben-Khemis

21 (1975), no 2, p. 108-109

Blais, Raymond. Vocabulaire libre en indexation, par Raymond Blais et Yves Courrier. (Madeleine Laliberté)

25 (1979), no 2, p. 107-108

Blasselle, Bruno. La Bibliothèque nationale, mémoire de l'avenir, par Bruno Blasselle et Jacqueline Melet-Sanson. (Jean-Rémi Brault)

$$
37 \text { (1991), no 2, p. 84-85 }
$$

Blazina, Vesna. Thésaurus : délinquance juvénile, par Vesna Blazina et Sophie Janik. (Lise Brunet)

28 (1982), no 1, p. $35-36$ 
Blouin, Louise. Répertoire des oeuvres de la littérature radiophonique québécoise, 1930-1970, par Pierre Pagé avec la collaboration de Renée Legris et Louise Blouin. (Jean-Pierre Chalifoux) 21 (1975), no 3, p. 171-172

Blouin, Nicole. Communication et relations publiques. (Céline R. Cartier)

19 (1973), no 1, p. 43-45

(Blouin-Cliche, Odette). Rinfret, Edouard G. Le théâtre canadien d'expression française; répertoire analytique des origines à nos jours

$$
22 \text { (1976), no 2, p. } 94
$$

(Boilard, Gilberte). Robert, Paul. Dictionnaire alphabétique et analogique de la langue française, $2 e$ éd.

32 (1986), no 1-2, p. 53-54

(Boilard, Thérèse). Castonguay, André. Lire à "Bordeaux", sous la direction de André Castonguay.

20 (1974), no 2, p. 105-106

(Boily, Denis). Centrale des bibliothèques. Centre de bibliographie. Périodiques pour les collèges

21 (1975), no 2, p. 106-108

Boily, Robert. Le Québec en chiffres de 1850 à nos jours, par Gérald Bernier et Robert Boily. (Roger de la Sablonnière)

35 (1989), no 3, p. 121-123

Boily, Robert. Le Québec en transition : 1760-1867 : bibliographie thématique, par Gérald Bernier et Robert Boily. (Monique Mailloux)

34 (1988), no 4, p. $159-162$

(Boisvert, Michel). Pagé, Pierre. Répertoire des dramatiques québécoises à la télévision, 1952-1977, par Pierre Pagé et Renée Legris.

24 (1978), no 2, p. 91

Boivin, Aurélien. Le conte littéraire québécois au XIXe siècle : essai de bibliographie critique et analytique. (Pierre Giguère)

22 (1976), no 3, p. 149-150

Boivin, Henri-Bernard. Les ouvrages de référence du Québec : supplément anaIytique. (Jean-Marie Brière)

32 (1986), no 1-2, p. 63-64

Boncenne, Pierre

voir La bibliothèque idéale
Bonin, Claude-André et al. Lexique d'information documentaire $=$ Lexicon of Information and Documentation. (Gaston Bernier)

32 (1986), no 4, p. 160-161

Bonnefoy, Claude. Dictionnaire de littérature française contemporaine, par Claude Bonnefoy, Tony Cartano et Daniel Oster. (Patrick Coppens)

24 (1978), no 3, p. 146-148

(Bonnelly, Claude). Hurtubise, Rolland. Informatique et information; la conception des systèmes d'information; les banques de données

25 (1979), no 2, p. 103-105

Bonnet, Alain. L'intelligence artificielle promesse et réalités. (Sophie Janik)

31 (1985), no 4, p. 171-172

(Bonville, Jean de). Antennes, la revue québécoise des communications. 1976-

22 (1976), no 3, p. 145-146

(Bonville, Jean de). Association pour l'avancement des sciences et des techniques de la documentation. Travaux du 10 e congrès tenu à Montréal du 27 au 30 octobre 1983. Au-delà de l'information : la communication

32 (1986), no 3, p. 97-98

(Bonville, Jean de). Bâtir l'avenir. Les communications au Québec : recherche et développement. Bilan et perspectives

29 (1983), no 2, p. 84-88

(Bonville, Jean de). Communication et Information. 1975-(Périodique)

$$
22 \text { (1976), no 3, P. 145-146 }
$$

(Bonville, Jean de). Faurie, Albertine. La pédagogie de la documentation dans le contexte de l'audiovisuel et des massmédia

$$
27 \text { (1981), no 2, p. 86-87 }
$$

(Bonville, Jean de). Multi-Réso Inc. La presse écrite au Québec. Bilan et prospective

$$
25 \text { (1979), no 3, p. 157-161 }
$$

(Bonville, Jean de). Multi-Réso Inc. Une agence de presse au Québec? Nécessité, utilité et viabilité économique (Rapport)

25 (1979), no 3 , p. $157-161$
(Bonville, Jean de). Québec. Ministère des Communications. Direction générale du Développement et des Politiques. De la précarité de la presse ou le citoyen menacé. Rapport du comité de travail sur la concentration de la presse écrite

25 (1979), no 3, p. 157-161

Bonville, Jean de. Bibliocom 1976; bibliographie internationale de la documentation en langue française sur la communication. (Marc Waller)

26 (1980), no 2, p. 117-118

Bonville, Jean de. Bibliocom 1977; bibliographie internationale de la documentation en langue française sur la communication. (Marc Waller)

26 (1980), no 2, p. 117-118

Bonville, Jean de. Introduction aux ouvrages généraux de référence sur la communication et les mass media. (Marc Alain)

22 (1976), no 1, p. 49-50

(Bosa, Réal). Olier, J. H. d'. La planification des infrastructures nationales de documentation, de bibliothèques et d'archives, par J. H. d'Olier et B. Delmas

22 (1976), no 1, p. 50-51

(Bosa, Réal). Thibault, Danielle. Guide de rédaction bibliographique

37 (1991), no 3, p. 122

(Bouchard, Claude). L'enfant, l'image et le récit. Ouvrage collectif sous la direction de Denise Escarpit

25 (1979), no 3, p. 169-171

(Boucher, Alain). Bibliothèque nationale du Canada. Groupe de travail sur le catalogue collectif canadien. Rapport final soumis au directeur général de la Bibliothèque nationale

24 (1978), no 1, p. 44-45

(Boucher, Alain). Bibliothèque nationale du Canada. Groupe de travail sur le catalogue collectif canadien. Sous-groupe de travail sur les catalogues collectifs de publications en série. Rapport

24 (1978), no 1, p. 44-45

(Boucher, Denis). Choix de titres d'ouvrages canadiens en langue française 1974

20 (1974), no 3, p. 167 
(Boucher, Denis). Jolicoeur, Louis-Philippe. Revues d'analyse et d'indexation. Répertoire annoté des revues d'analyse et d'indexation de la collection de la $\mathrm{Bi}$ bliothèque de l'Université Laval

23 (1977), no 3, p. 174-175

Bourgault, Jacques. Guide de recherche documentaire en matière de publications parlementaires et gouvernementales du Québec. (Gaston Bernier)

31 (1985), no 1 , p. $40-41$

Bourget, Manon. L'indispensable en documentation; les outils de travail, par Manon Bourget, Robert Chiasson et MarieJosée Morin. (Léonard Nadeau)

37 (1991), no 1, p. $43-44$

(Bourneuf, Denyse). Mignault, Marcel. Lise et Bruno dans l'univers des livres

23 (1977), no 3, p. 173-174

Bourret, Annie. Guerre, paix et désarmement. Bibliographie thématique en langue française, par Erik Poole et Annie Bourret. (Rénald Buteau)

36 (1990), no 3, p. 108-109

Bourret, Annie. Index sur la guerre et la paix : index relationnel de mots-clés pour SONJA, banque de données informatisées sur la guerre, la paix et le désarmement en langue française, par Annie Bourret et Érik Poole. (Rénald Buteau)

36 (1990), no 3, p. 108-109

Bourron, Yves. Audiovisuel, pédagogie et communication. (Claire Meunier)

28 (1982), no 4, p. $169-170$

Bousso, Amadou. La formation des bibliothécaires et le développement des bibliothèques universitaires en Afrique. (Jean-Luc Fortin)

29 (1983), no 2, p. 80-82

(Bouthillette, Jean). Dubuc, Pierrette. Thésaurus : Enfance inadaptée, par Pierrette Dubuc, Lise Thibodeau-Brunet et Luc-Louis Lecompte

27 (1981), no 2, p. $82-83$

(Brault, Jean-Rémi). A l'écoute de l'oeil : les collections iconographiques et les bibliothèques

35 (1989), no 4, p. $193-194$

(Brault, Jean-Rémi). Alain, Jean-Marc. Pour une gestion adaptée des services documentaires

36 (1990), no 1, p. $37-39$
(Brault, Jean-Rémi). Arbido-B. 33 (1987), no 1, p. $31-32$

(Brault, Jean-Rémi). Arbido-R 33 (1987), no 1, p. 31-32

(Brault, Jean-Rémi). Beaudiquez, Marcelle. Inventaire général des bibliographies nationales rétrospectives $=$ Retrospective National Bibliographies : An International Directory

33 (1987), no 3, p. 104-105

(Brault, Jean-Rémi). Bernier, Silvie. Du texte à l'image : le livre illustré au Québec

37 (1991), no 3, p. 125

(Brault, Jean-Rémi). Bibliothèque de France, bibliothèque ouverte

37 (1991), no 3, p. 121-122

(Brault, Jean-Rémi). Bibliothèque nationale du Canada. Groupe de travail sur le service de bibliothèque aux handicapés. Rapport

24 (1978), no 1, p. 41-43

(Brault, Jean-Rémi). Blasselle, Bruno. La Bibliothèque nationale, mémoire de l'avenir, par Bruno Blasselle et Jacqueline Melet-Sanson

37 (1991), no 2, p. $84-85$

(Brault, Jean-Rémi). Cahart, Patrice. Propositions pour une grande bibliothèque, par Patrice Cahart et Michel Melot

36 (1990), no 3, p. 111-112

(Brault, Jean-Rémi). Cahart, Pierre. Le livre français a-t-il un avenir ?

35 (1989), no 2, p. $64-66$

(Brault, Jean-Rémi). Cirkovic-Stanojlovic, Ljiljana. La restauration mineure des documents sur papier; initiation pratique, par Ljiljana Cirkovic-Stanojlovic en collaboration avec Robert Chiasson

34 (1988), no 1 , p. 37

(Brault, Jean-Rémi). Classification décimale universelle (CDU)

34 (1988), no 1 , p. $38-39$

(Brault, Jean-Rémi). Colloque sur l'accès aux publications au Canada, Québec, 1986. L'accès aux publications au Canada 35 (1989), no 1, p. 27

(Brault, Jean-Rémi). Desjardins, Marc. Bibliographie de la Gaspésie 35 (1989), no 1, p. 31
(Brault, Jean-Rémi). Ducharme, Jacques Guide provisoire des Archives judiciaires du district de Montréal

33 (1987), no 3, p. 107-108

(Brault, Jean-Rémi). Eco, Umberto. De bibliotheca

32 (1986), no 4, p.

(Brault, Jean-Rémi). Figuier, Richard dirigé par. La bibliothèque, miroir de l'âme, mémoire du monde

38 (1992), no 4, p. 216-217

(Brault, Jean-Rémi). Janik, Sophie. Bibliographie québécoise sur les personnes handicapées, établie par Sophie Janik avec la collaboration de Edward A Collister et Lise Brunet

35 (1989), no 1, p. 29-30

(Brault, Jean-Rémi). Jutras, Pierre sous la dir. de. Annuaire du cinéma québécois 1990

38 (1992), no 4, p. $215-216$

(Brault, Jean-Rémi). L'édition du livre populaire : études sur les Éditions Édouard Garand, de l'Étoile, Marquis, Granger Frères, rassemblées et présentées par Jacques Michon

34 (1988), no 4, p. 159

(Brault, Jean-Rémi). La bibliothèque idéale, sous la direction de Pierre Boncenne

35 (1989), no 3, p. 125-126

(Brault, Jean-Rémi). Lafortune, Hélène et al. Parchemin s'explique...; guide de dépouillement des actes notariés du Québec ancien

35 (1989), no 3, p. 126

(Brault, Jean-Rémi). Lagrave, Jean-Paul de. Fleury Mesplet (1734-1794); diffuseur des lumières au Québec

32 (1986), no 1-2, p. 55-56

(Brault, Jean-Rémi). Lajeunesse, Marcel Les bibliothèques des collèges d'enseignement général et professionnel du Québec : étude de leur évolution, (19691983)

32 (1986), no 1-2, p. 54-55

(Brault, Jean-Rémi). Lamothe, Madeleine. Archives des femmes du Québec; guide sommaire. Assistée de Ghislaine Fecteau et Pierrette Lalancette

37 (1991), no 2, p. 85-86 
(Brault, Jean-Rémi). Libère, François. Le livre, mutations actuelles

36 (1990), no 4, p. 141

(Brault, Jean-Rémi). Livre et lecture au Québec (1800-1850), sous la direction de Claude Galarneau et Maurice Lemire

34 (1988), no 4 , p. $159-160$

(Brault, Jean-Rémi). Mennie-de Varennes, Kathleen. Bibliographie annotée d'ouvrages généalogiques au Canada = Annotated Bibliography of Genealogical Works in Canada

33 (1987), no 4, p. 138

(Brault, Jean-Rémi). Mittermeyer, Diane. Les bibliothèques publiques canadiennes de 1979 à 1983 : analyse comparative de certaines données statistiques

33 (1987), no 4, p. $139-140$

(Brault, Jean-Rémi). Office des personnes handicapées du Québec. 300 documents audiovisuels sur les personnes handicapées

35 (1989), no 1 , p. $29-30$

(Brault, Jean-Rémi). Répertoire des centres de documentation et des bibliothèques spécialisées et de recherche, 1992

38 (1992), no 4 , p. $209-210$

(Brault, Jean-Rémi). Roberge, Michel. L'expertise québécoise en gestion des documents administratifs; bibliographie thématique et chronologique 1962-1987

34 (1988), no 3, p. 113

(Brault, Jean-Rémi). Sénécal, André. A Reader's Guide to Québec Studies

36 (1990), no 2, p. 77-78

(Brault, Jean-Rémi). Thériault, Michel. Les instituts de vie consacrée au Canada depuis les débuts de la Nouvelle-France jusqu'à aujourd'hui; notes historiques et références. The Institutes of Consacred Life in Canada from the Beginning of the New France up to the Present; Historical Notes and References

$$
27 \text { (1981), no 3, p. } 132
$$

Brault, Jean-Rémi. Vers une planification des services québécois d'information documentaire. Textes déposés lors de la Table ronde, 5 mai 1978, présentés par Jean-Rémi Brault. (Denis Simard)

26 (1980), no 3, p. 169-172
Breton, Jean et al. Guide pratique d'indexation en vocabulaire libre. (Danielle Malette)

30 (1984), no 2, p. $63-64$

Bridging the Gap between Theory and Pratice

voir Théorie et pratique dans l'enseignement des sciences de l'information. Bridging the Gap between Theory and Practice

(Brière, Jean-Marie). Boivin, Henri-Bernard. Les ouvrages de référence du Québec: supplément analytique

32 (1986), no 1-2, p. 63-64

Brière, Jean-Marie. L'information gouvernementale au Québec : bibliographie analytique. (Daniel Allaire)

25 (1979), no 1, p. $48-49$

Brochures québécoises 1764-1972 voir Hamelin, Jean

Brodeur, Raymond et al. Les catéchismes au Québec, 1702-1963. (Gilles Gallichan)

37 (1991), no 3, p. 122-123

(Brougère, Gilles). Garon, Denise. La classification des jeux et des jouets - Le système ESAR

$$
33 \text { (1987), no 1, p. } 30-31
$$

Brown, Barbara E. rédactrice. Sources d'informations économiques et commerciales canadiennes / Canadian Business and Economics: A guide to Sources of Information. (Maurice Saint-Germain) 23 (1977), no 1, p. 47-48

Brunet, Lise Thibodeau voir Thibodeau-Brunet, Lise

(Brunet, Lise). Bibliothèque nationale du Québec. Radar, répertoire analytique d'articles de revues du Québec 19 (1973), no 2, p. $91-93$

(Brunet, Lise). Blazina, Vesna. Thésaurus : délinquance juvénile, par Vesna Blazina et Sophie Janik

28 (1982), no 1, p. 35-36

(Brunet, Lise). Bureau international du travail. ILO Thesaurus - Thésaurus B.I.T. Tesauro O.I.T.

22 (1976), no 4, p. 203-204

(Brunet, Lise). Centrale des bibliothèques. Periodex, index analytique de périodiques de langue française

19 (1973), no 2, p. $91-93$
Buchinski, Edwin J. Le contexte de l'interconnexion dans le cadre de l'élaboration d'un réseau bibliographique national. (Jean Thibault)

27 (1981), no 3, p. 128

(Bull, Jerry). Deschâtelets, Gilles. Enquête sur les services de téléréférence au Canada.

27 (1981), no 2, p. 83-84

(Bull, Jerry). France-actualité, index de la presse écrite française

25 (1979), no 3, p. 162-164

(Bull, Jerry). Index de l'actualité vue à travers la presse écrite

23 (1977), no 2, p. 114-116

Bulletin du livre. no 1- 1958- . . (Jacques Paradis)

19 (1973), no 3, p. 134-136

Bulletin signalétique d'information administrative. (Gaston Bernier)

30 (1984), no 1, p. $28-29$

Buono, Yolande. Catalogue collectif des impressions québécoises. 1764-1820, par Milada Vlach et Yolande Buono. (Gilles Gallichan)

30 (1984), no 4, p. $142-143$

Buono, Yolande. Laurentiana parus avant 1821, par Milada Vlach, avec la collaboration de Yolande Buono. (John E. Hare)

23 (1977), no 4, p. $215-216$

Bureau de la statistique du Québec voir Québec. Bureau de la statistique du Québec

Bureau international du travail. ILO Thesaurus - Thésaurus B.I.T. - Tesauro O.I.T. (Lise Brunet)

22 (1976), no 4, p. 203-204

(Burton, Françoise). Fontaine, France. Principes directeurs pour la rédaction d'objectifs d'apprentissage en bibliothéconomie, en sciences de l'information et en archivistique

35 (1989), no 4 , p. $190-191$

(Busque, Claude). Gaudette, Pierre. La répartition du budget des collections au Service des bibliothèques de l'Université de Sherbrooke

32 (1986), no 3, p. $99-100$ 
(Busque, Claude). Such, Marie-France. Initiation à la bibliographie scientifique, par Marie-France Such et Dominique Perol

35 (1989), no 1, p. 28-29

(Buteau, Rénald). Bourret, Annie. Index sur la guerre et la paix : index relationnel de mots-clés pour SONJA, banque de données informatisées sur la guerre, la paix et le désarmement en langue française, par Annie Bourret et Érik Poole

36 (1990), no 3, p. 108-109

(Buteau, Rénald). Macrothésaurus pour le traitement de l'information relative au développement économique et social ( $4 \mathrm{e}$ éd.)

$$
37 \text { (1991), no 4, p. } 167
$$

(Buteau, Rénald). Poole, Erik. Guerre, paix et désarmement. Bibliographie thématique en langue française, par Érik Poole et Annie Bourret

36 (1990), no 3, p. 108-109

(Buteau, Rénald). Szpakowska, JaninaKlara. Jeunes gens : thésaurus; essai de systématisation des connaissances spécifiques à la classe d'âge 13-25 ans

35 (1989), no 3, p. 123-124

\section{C}

Cahart, Patrice. Propositions pour une grande bibliothèque, par Patrice Cahart et Michel Melot. (Jean-Rémi Brault)

36 (1990), no 3, p. 111-112

Cahart, Pierre. Le livre français a-t-il un avenir? (Jean-Rémi Brault)

35 (1989), no 2, p. $64-66$

Cain, Julien. Le livre français : hier, aujourd'hui, demain. Ouvrage collectif sous la direction de Julien Cain, Robert Escarpit, Henri-Jean Martin. (Jean-Louis Roy)

20 (1974), no 1, p. 44-45

Calvé-Woodrough, Lise. Recueil d'exemples illustrant les Règles de catalogage anglo-américaines. Edition provisoire sous la direction de Lise CalvéWoodrough et Michel Faubert. Avec la collaboration de Louise Gagnon (et autres). (Léonard Nadeau)

21 (1975), no 4, p. 231-232
Campbell, Henry L. Le développement des systèmes et des services de bibliothèques publiques : guide pour l'organisation des systèmes nationaux et régionaux de bibliothèques publiques en tant que composantes de la planification générale des services nationaux d'information. (Marcel Lajeunesse)

31 (1985), no 2, p. 75-76

Le Canada français et sa littérature de Jeunesse

voir Potvin, Claude

Canada. Archives publiques. Plan de conservation et d'élimination de documents. (Yvon Papillon)

28 (1982), no 3, p. 132-133

Canada. Bibliothèque nationale du Canada voir Bibliothèque nationale du Canada

Canada. Comité des systèmes unifiés de bibliothèque. Groupe de travail sur la mesure de la performance. Mesure de la performance dans les bibliothèques fédérales : un guide. Performance Measurement in Federal Libraries : A Handbook. (Gaétan Drolet)

$$
27 \text { (1981), no 2, p. 84-86 }
$$

Canada. Groupe de travail sur la normalisation du catalogage. Normalisation du catalogage. (Raymonde Couture-Lafleur)

20 (1974), no 3 , p. $169-170$

Canada. Statistique Canada. Division de l'éducation, des sciences et de la culture. Section des beaux-arts et des médias: Literary and Journalistic Awards in Canada I Les prix de littérature et de journalisme au Canada, 1923-1973. (Jean-Louis Fortier et Richard Giguère)

23 (1977), no 2, p. $117-120$

Canadian Business and Economics : A Guide to Sources of Information

voir Brown, Barbara $\mathrm{E}$.

Canadian Guide to Rare Books

voir Gauvin, Daniel

Canadian Library Yearbook = Annuaire des bibliothèques canadiennes. (Gaston Bernier)

31 (1985), no 4, p. 167-168
Cantin, Pierre. Bibliographie de la critique de la littérature québécoise et canadiennefrançaise dans les revues canadiennes (1974-1978), par René Dionne et Pierre Cantin. (Claude Fournier)

37 (1991), no 2, p. 83-84

Cardinal, Robert. Les bibliothèques canadiennes à l'ère de l'automatisation : synthèse bibliographique 1970-72, par Colette Rivet-Panaccio, Amal Awad et Robert Cardinal. (Marcel Fontaine)

19 (1973), no 2, p. 93-94

(Caron, Gilles). Cossette, André. Humanisme et bibliothèques : essai sur la philosophie de la bibliothéconomie

22 (1976), no 4, p. 197-199

(Caron, Raymond). Dubuc, Pierrette. Thésaurus : Enfance inadaptée, par Pierrette Dubuc, Lise Thibodeau-Brunet et Luc-Louis Lecompte

27 (1981), no 2, p. 82-83

Carroll, Frances Laverne. Directives pour la conception et l'organisation de médiathèques scolaires, par Frances Laverne Carroll et Patricia F. Beilke. (Paulette Bernhard)

29 (1983), no 1, p. $43-44$

Cartano, Tony. Dictionnaire de littérature française contemporaine, par Claude Bonnefoy, Tony Cartano et Daniel Oster. (Patrick Coppens)

24 (1978), no 3, p. 146-148

(Cartier, Céline R.). Blouin, Nicole. Communication et relations publiques 19 (1973), no 1, p. $43-45$

(Cartier, Céline). Miquel, André. Les bibliothèques universitaires. Rapport au ministre d'État, ministre de l'Éducation nationale, de la Jeunesse et des Sports

36 (1990), no 2, p. 75-77

Carto-03 : Répertoire cartobibliographique sur la région de Québec, compilé par Yves Tessier avec la collaboration de Jacques Martinez et la participation de Louise Lavoie. (Yves Tessier)

33 (1987), no 2, p. 74-76

Castonguay, André. Lire à "Bordeaux", sous la direction de André Castonguay. (Thérèse Boilard)

20 (1974), no 2, p. 105-106 
Castonguay, André. Une bibliothèque pour Montréal; rapport préléminaire sur la possibilité d'établir une bibliothèque publique à la Place Desjardins, sous la direction de André Castonguay, par JeanMarc Alain (et autres). (Jacques Panneton)

20 (1974), no 1, p. $42-43$

Catalogue collectif des impressions québécoises. 1764-1820

voir Vlach, Milada

Catalogue collectif des manuscrits des archives canadiennes

voir Archives publiques du Canada

Catalogue collectif des périodiques de la région de Montréal

voir Union List of Serials in the Montreal Area $=$ Catalogue collectif des périodiques de la région de Montréal, 1986

Catalogue de la bibliothèque de LouisJoseph Papineau

voir Lemoine, Roger

Catalogue de livres français 1972 / French Book Catalogue. (Anna Rovira)

20 (1974), no 3, p. 167-168

Catalogue des films

voir Ville de Montréal. Service des activités culturelles. Division des bibliothèques

Catalogue des manuscrits

voir Bilbiothèque nationale du Québec

Catalogue des vidéocassettes

voir Ville de Montréal. Service des activités culturelles. Division des bibliothèques

Les catéchismes au Québec, 1702-1963 voir Brodeur, Raymond

Cau, Ignace. L'édition au Québec de 1960 à 1977. (Marcel Lajeunesse)

28 (1982), no 3, p. 127-128

(Caya, Marcel). Québec. Assemblée nationale. Débats de l'Assemblée législative, 1867-1878, 1893-1901

30 (1984), no 2, p. $67-68$

CEGEP de Trois-Rivières. Films et vidéocassettes - Catalogue 1986. 4e éd. (Louise de Chevigny)

32 (1986), no 4, p. 157-158

Centrale des bibliothèques. Centre de bibliographie. Périodiques pour les collèges. (Denis Boily)

21 (1975), no 2, p. 106-108
Centrale des bibliothèques. Periodex, index analytique de périodiques de langue française. (Lise Brunet)

19 (1973), no 2, p. $91-93$

Centre d'études en enseignement du Canada. L'inter Atlas : les ressources du Québec et du Canada. (Pierre Lépine)

34 (1988), no 3, p. 110-111

Centre de recherche en civilisation canadienne-française

voir Université d'Ottawa. Centre de recherche en civilisation canadienne-française

Les centres de ressources éducatives dans l'enseignement secondaire : exploration de la diversité des modèles, des situations et des problèmes locaux

voir Bergeron, Christian

\section{CERBIQ}

voir Québec. Comité d'étude d'un réseau des bibliothèques québécoises pour le traitement en coopération de leur documentation

Ces lectures sauvages; panoramique sur le service de bibliothèque aux jeunes adultes voir Szpakowska, Janina-Klara

(Chabot, Jean-Pierre). Des livres et des jeunes (vol.1, no 1-)

26 (1980), no 2, p. 119-122

(Chabot, Jean-Pierre). Lemire, Maurice, éd. Dictionnaire des oeuvres littéraires du Québec. Tome 1: des origines à 1900

$$
25 \text { (1979), no 1, p. } 53-56
$$

(Chabot, Jean-Pierre). Lurelu : bulletin d'information sur la littérature de jeunesse (vol.1, no 1-)

26 (1980), no 2, p. 119-122

Chaintreau, Anne-Marie. Drôles de bibliothèques... : le thème de la bibliothèque dans la littérature et le cinéma, par AnneMarie Chaintreau et Renée Lemaître. (Jacques Paradis)

$$
37 \text { (1991), no 1, p. } 44-46
$$

(Chalifoux, Jean-Pierre). Pagé, Pierre. Répertoire des oeuvres de la littérature radiophonique québécoise, 1930-1970, par Pierre Pagé, avec la collaboration de Renée Legris et Louis Blouin

$$
21 \text { (1975), no 3, p. 171-172 }
$$

(Chalifoux, Jean-Pierre). Québec. Bibliothèque nationale du Québec. Bibliographie de bibliographies québécoises

26 (1980), no 1, p. 51-53

(Chamberot, Robert). Allard, Yvon. Écrits sur l'avenir

29 (1983), no 1, p. $45-46$

(Chantal, Jean de). Amoikon, Michel K Structures, organisation et animation culturelle dans les bibliothèques en Côte d'Ivoire

22 (1976), no 4, p. 199-203

(Chantal, Jean de). Association internationale pour le développement de la documentation, des bibliothèques et des archives en Afrique. Conférence sur les problèmes de la planification et de l'organisation des archives, bibliothèques et centres de documentation en Afrique, Abidjan, 11-17 septembre 1972

22 (1976), no 4, p. 199-203

(Chantal, Jean de). Lalande-Isnard, F. Les bibliothèques dans trois pays de l'ouest africain (Guinée, Mali, Sierra Leone)

22 (1976), no 4, p. 199-203

Chantigny, Louis et al. Bernard Valiquette. (Claude Fournier)

32 (1986), no 1-2, p. 56-57

(Charbonneau, Hélène). Danset-Léger, Jacqueline. L'enfant et les images de la littérature enfantine

28 (1982), no 3 , p. $130-131$

(Charbonneau, Hélène). Tremblay, Ginette. Croc-enlivre. Rencontre avec les livres. Pour une animation du livre avec les 4-10 ans, par Ginette Tremblay et Pierrette Santerre

31 (1985), no 4, p. 168-169

Charbonneau, Hélène. Livres en langue française pour les jeunes. (Louisette Bergeron)

32 (1986), no 4, p. $158-160$

(Charette, Christiane). Barut, Catherine. Bibliothèques dans la rue. Une expérience des bibliothèques municipales de la Ville de Genève, par Catherine Barut et Isabelle Wenger

33 (1987), no 2, p. 71-72 
(Charette, Christiane). Szpakowska, Janina-Klara. Besoins en information et en services documentaires chez les jeunes de 14-19 ans

26 (1980), no 1, p. 53-55

Chartrand, Georges A. Livre, bibliothèque et culture québécoise. Mélanges offerts à Edmond Desrochers, sous la direction de Georges A. Chartrand. (Hubert Perron)

25 (1979), no 2, p. 101-103

(Chartrand, Georges-A.). Cormier, Normand. Maria Chapdelaine : évolution de l'édition 1913-1980. Equipe de recherche: Normand Cormier, responsable, et al.

27 (1981), no 2, p. 81-82

(Chartrand, Georges-A.). Université Laval. Bibliothèque. Service de catalogage. Index anglais-français des termes utilisés dans le Répertoire de vedettes-matière

19 (1973), no 4, p. 178-181

(Chartrand, Georges-A.). Université Laval. Bibliothèque. Service de catalogage. Répertoire de vedettes-matière (7e éd.)

19 (1973), no 4, p. 178-181

Chaumier, Jacques. Le traitement linguistique de l'information documentaire : l'analyse documentaire. (Suzanne Bertrand-Gastaldy)

24 (1978), no 3, p. 148-151

Chaumier, Jacques. Travail et méthodes du/de la documentaliste (Marylène Le Deuff)

28 (1982), no 1, p. $33-35$

Chauveinc, Marc. Le réseau bibliographique informatisé et l'accès au document. (Jean-Pierre Côté)

29 (1983), no 1 , p. $46-48$

Cherns, Jack J. Accessibilité et utilisation des publications officielles dans les bibliothèques. (Gaston Bernier)

32 (1986), no 1-2, p. 60-61

Chevalier, Brigitte. Méthodologie d'utilisation d'un centre de documentation. (Jean-Luc Roy)

28 (1982), no 1 , p. $39-40$

(Chevigny, Louise de). CEGEP de TroisRivières. Films et vidéocassettes - Catalogue 1986. 4e éd.

32 (1986), no 4, p. 157-158
(Chevigny, Louise de). Ville de Montréal. Service des activités culturelles. Division des bibliothèques. Catalogue des films 32 (1986), no 4, p. 157-158

(Chevigny, Louise de). Ville de Montréal. Service des activités culturelles. Division des bibliothèques. Catalogue des vidéocassettes

$$
32 \text { (1986), no 4, p. 157-158 }
$$

Chiasson, Robert. L'indispensable en documentation; les outils de travail, par Manon Bourget, Robert Chiasson et MarieJosée Morin. (Léonard Nadeau)

37 (1991), no 1, p. $43-44$

Choix de titres d'ouvrages canadiens en langue française 1974. (Denis Boucher)

20 (1974), no 3, p. 167

Choix et acquisition des documents au Québec. Volume 1 : Principes et applications

voir Thériault, Michel

Choix Jeunesse. Jeux et Jouets 1981 (Jacqueline Thériault) 29 (1983), no 3 , p. $122-124$

Choix Jeunesse. Jeux et Jouets 1982 (Jacqueline Thériault) 29 (1983), no 3, p. 122-124

Choquette, Louisette Bergeron voir Bergeron-Choquette, Louisette

Les cinémas canadiens. Dossiers établis sous la direction de Pierre Véronneau. (Alain Julien)

25 (1979), no 3, p. 164-165

Cirkovic-Stanojlovic, Ljiljana. La restauration mineure des documents sur papier; initiation pratique, par Ljiljana CirkovicStanojlovic en collaboration avec Robert Chiasson. (Jean-Rémi Brault) 34 (1988), no 1, p. 37

Classe FC. Cadre de classification de l'histoire du Canada. (Paule RollandThomas et Marcel Lajeunesse) 23 (1977), no 2, p. 112-114

Classe PS 8000 . Cadre de classification de la littérature canadienne (2e éd.). (Nicole Genest et Denise Lebel-Paquin) 25 (1979), no 1, p. 50-51

Classification décimale de Dewey et index, établis par Melvil Dewey

voir Dewey, Melvil
Classification décimale universelle (CDU). (Jean-Rémi Brault)

34 (1988), no 1 , p. $38-39$

La classification des jeux et des jouets - Le systeme ESAR

voir Garon, Denise

Classification des publications gouvernementales du Québec

voir Filion, Louise

Clavel Jean-Pierre et al. L'évaluation des bibliothèques universitaires. (Clément Tremblay)

32 (1986), no 1-2, p. 58-59

Cliche, Odette Blouin

voir Blouin-Cliche, Odette

Collections de recherche des bibliothèques canadiennes. (Marguerite Densky)

20 (1974), no 2, p. 107-110

(Collister, Edward A.). Colloque sur la conservation des collections, Montréal, 1617 novembre 1989. Prévenir ou guérir?

36 (1990), no 4, p. 143-144

Colloque sur l'accès aux publications au Canada, Québec, 1986. L'accès aux publications au Canada. (Jean-Rémi Brault)

35 (1989), no 1, p. 27

Colloque sur la conservation des collections Montréal, 16-17 novembre 1989. Prévenir ou guérir? (Edward $A$. Collister) 36 (1990), no 4, p. 143-144

Colpron, Gilles. Dictionnaire des anglicismes. (Louis-Jean Rousseau) 32 (1986), no 1-2, p. 61-62

Comité consultatif du livre voir Québec. Comité consultatif du livre

Comité des systèmes unifiés de bibliothèque. Groupe de travail sur la mesure de la performance

voir Canada. Comité des systèmes unifiés de bibliothèque. Groupe de travail sur la mesure de la performance

Commission de coordination de la documentation administrative voir France. Commission de coordination de la documentation administrative

Commissions royales provinciales et commissions d'enquêtes : bibliographie sélective voir Maillet, Lise 
Communication et information. 1975(Périodique). (Jean de Bonville)

$$
22 \text { (1976), no 3, p. 145-146 }
$$

Communication et relations publiques voir Blouin, Nicole

Comptes rendus

voir Conférence nationale sur l'état de la bibliographie au Canada

Conception et gestion des systèmes documentaires

voir Van Slype, Georges

Conférence nationale sur l'état de la bibliographie au Canada, Vancouver, les 22, 23 et 24 mai 1974. Comptes rendus. (V. Alexandre Jonynas)

24 (1978), no 2, p. 92-95

Conférence sur les problèmes de la planification et de l'organisation des archives, bibliothèques et centres de documentation en Afrique, Abidjan, 11-17 septembre 1972

voir Association internationale pour le développement de la documentation, des bibliothèques et des archives en Afrique

Connaissance et formation par le livre et l'audiovisuel. no.1- 1973- . (Jacques Paradis)

$$
20 \text { (1974), no 1, p. } 45-47
$$

Conseil du statut de la femme

voir Québec (Province). Conseil du statut de la femme

(Constantin, Jacques). Roy, Jean-Louis. Edouard-Raymond Fabre, libraire et patriote canadien, 1799-1854

21 (1975), no 2, p. 105-106

Le conte littéraire québécois au XIXe siècle : essai de bibliographie critique et analytique voir Boivin, Aurélien

Le contexte de l'interconnexion dans le cadre de l'élaboration d'un réseau bibliographique national

voir Buchinski, Edwin J.

Contribution à la bibliographie nationale du Rwanda

voir Lévesque, Albert

Contribution to the National Bibliography of Rwanda : 1965-1970. Contribution à la bibliographie nationale du Rwanda voir Lévesque, Albert
(Coppens, Patrick). Bonnefoy, Claude. Dictionnaire de littérature française contemporaine, par Claude Bonnefoy, Tony Cartano et Daniel Oster

$$
24 \text { (1978), no } 3 \text {, p. } 146-148
$$

(Coppens, Patrick). Hamel, Réginald. Dictionnaire pratique des auteurs québécois, par Réginald Hamel, John Hare et Paul Wyczynski

23 (1977), no 2, p. 109-112

Corbeil, Jean-Claude. Dictionnaire thématique visuel. (Gaston Bernier)

33 (1987), no 3, p. 105-107

(Cormier, Normand). Gagné, Marc. Gilles Vigneault : bibliographie descriptive et critique, discographie, filmographie, iconographie, chronologie

24 (1978), no 4, p. 203-205

Cormier, Normand. Les ouvrages de référence du Québec. Supplément 19671976, par Suzanne Lauzier et Normand Cormier. (V. Alexandre Jonynas)

22 (1976), no 2, p. 91-93

Cormier, Normand. Maria Chapdelaine : évolution de l'édition 1913-1980. Equipe de recherche : Normand Cormier, responsable, et al. (Georges-A. Chartrand) 27 (1981), no 2, p. 81-82

(Corriveau, Conrad). Lamy-Rousseau, Françoise. Traitement automatisé des documents multimedia avec les systèmes ISBD unifié, Lamy-Rousseau et PRECIS. Proposition SILP

21 (1975), no 4, p. 229-231

Cossette, André. Humanisme et bibliothèques: essai sur la philosophie de la bibliothéconomie (Gilles Caron)

22 (1976), no 4, p. 197-199

Cossette, Claude. La publicité en action : comment élaborer une campagne de publicité ou ce qui se passe derrière les murs d'une agence, par Claude Cossette et René Déry. (Yves Tessier)

34 (1988), no 1, p. $39-40$

(Côté, Nicole). ASTED. Commission des bibliothèques spécialisées et de recherche. Répertoire des outils documentaires dans les centres de documentations

27 (1981), no 3, p. 126-127

(Côté, Nicole). Gourd, Benoit-Beaudry. Bibliographie de l'Abitibi-Témiscamingue

24 (1978), no 1, p. $45-47$
Côté, Pierre A. Problèmes de droit d'auteur en éducation, par Christian Vincke. Avec la collaboration de Pierre $A$. Côté et Victor Nabhan. (Claudio Antonelli)

25 (1979), no 1, p. $49-50$

(Côté, Jean-Pierre). Chauveinc. Marc. Le réseau bibliographique informatisé et l'accès au document

29 (1983), no 1, p. $46-48$

(Cotnam, Jacques). Lamonde, Yvan. La librairie et l'édition à Montréal, 1776-1920

38 (1992), no 4, p. 212-213

Coulaud, Philippe. La télématique documentaire à l'épreuve. Usages et usagers des bases de données à la BPI. (Gilles Deschâtelets)

30 (1984), no 1, p. 32

(Coulombe, Victor). Lévesque, Albert Contribution to the National Bibliography of Rwanda : 1965-1970. Contribution à la bibliographie nationale du Rwanda

26 (1980), no 3, p. 173-177

Courrier, Yves. Vocabulaire libre en indexation, par Raymond Blais et Yves Courrier. (Madeleine Laliberté)

25 (1979), no 2, p. 107-108

(Couture, Carol). Walne, Peter et al. Dictionary of Archival Terminology $=$ Dictionnaire de terminologie archivistique. English and French with equivalent in Dutch, German, Italian, Russian and Spanish

32 (1986), no 3, p. 101-102

Couture, Carol, Les archives au XXe siècle : une réponse aux besoins de l'administration et de la recherche, par Carol Couture et Jean-Yves Rousseau. (Baïla Wane)

30 (1984), no 1 , p. $33-35$

(Couture-Lafleur, Raymonde). Canada. Groupe de travail sur la normalisation du catalogage. Normalisation du catalogage

20 (1974), no 3, p. 169-170

Couty, Daniel. Dictionnaire des littératures de langue française, par Jean-Pierre de Beaumarchais, Daniel Couty et Alain Rey. (Pierre Giguère)

31 (1985), no 2, p. 74-75

Croc-en-livre. Rencontre avec les livres Pour une animation du livre avec les 4-10 ans

voir Tremblay, Ginette 
D

d'Olier, J. H.

voir Olier, J. H. d'

Dagenais, Gérard. Dictionnaire des difficultés de la langue française au Canada. (Louis-Jean Rousseau)

32 (1986), no 1-2, p. 61,63

Danset-Léger, Jacqueline. L'enfant et les images de la littérature enfantine. (Hélène Charbonneau)

28 (1982), no 3, p. 130-131

(Daoust, Danièle). Raiffaud, Joël. La gestion documentaire, par Joël Raiffaud et associés

30 (1984), no 4, p. 144-145

Day, Mario. Accès aux publications gouvernementales québécoises et canadiennes : index permuté. (Gilbert Lefebvre)

25 (1979), no 3, p. 161-162

de Beaumarchais, Jean-Pierre

voir Beaumarchais, Jean-Pierre de

De bibliotheca

voir Eco, Umberto

de Bonville, Jean

voir Bonville, Jean de

de Chantal, Jean

voir Chantal, Jean de

de Chevigny, Louise

voir Chevigny, Louise de

de Grolier, Eric

voir Grolier, Eric de

De la précarité de la presse ou le citoyen menacé. Rapport du comité de travail sur la concentration de la presse écrite voir Québec. Ministère des Communications. Direction générale du Développement et des Politiques

de la Sablonnière, Roger

voir Sablonnière, Roger de la

de Lagrave, Jean-Paul

voir Lagrave, Jean-Paul de

de Varennes, Kathleen Mennie

voir Mennie-de Varennes, Kathleen

Débats de l'Assemblée législative, 18671878, 1893-1901

voir Québec. Assemblée nationale
Decroux-Masson, Annie. Papa lit, maman coud; les manuels scolaires en bleu et rose. (Noëlle Guilloton)

$$
26 \text { (1980), no 2, p. } 123
$$

Delmas, B. La planification des infrastructures nationales de documentation, de bibliothèques et d'archives, par J. H. d'Olier et $B$. Delmas. (Réal Bosa)

$$
22 \text { (1976), no 1, p. } 50-51
$$

(Delobel, Patrick). Une invitation : la bibliothèque scolaire mise à profit. Réalisation, Anne Bernier

$$
34 \text { (1988), no 3, p. 109-110 }
$$

(Delorme, Silvie). Herzhaft, Gérard. Pour une médiathèque : guide de l'audiovisuel à l'usage des candidats aux concours et examens des bibliothèques

$$
29 \text { (1983), no 3, p. 120-121 }
$$

(Demers, Jacques). Le dictionnaire du cinéma québécois, sous la direction de Michel Coulombe et Marcel Jean

$$
35 \text { (1989), no 2, p. 66-67 }
$$

(Demers, Jacques). Neale, Colin. Projet d'établissement d'un système d'information et de distribution pour les produits audiovisuels canadiens. Proposed Course of Action to Implement an Information/Distribution System for Canadian Audio-Visual Products

$$
24 \text { (1978), no 4, p. 205-208 }
$$

(Demers, Jacques). Rothwell, Hélène de F. A Proposal for a Selective, Annotated Catalogue of Canadian Produced NonPrint Materials. Research Report

$$
24 \text { (1978), no 4, p. 205-208 }
$$

Demers, Jacques. Gestion des documents audiovisuels dans les bibliothèques des collèges francophones du Québec. (Bernard Dumouchel)

$$
23 \text { (1977), no 4, p. 217-218 }
$$

(Densky, Marguerite). Collections de recherche des bibliothèques canadiennes

$$
20 \text { (1974), no 2, p. 107-110 }
$$

Déry, René. La publicité en action : comment élaborer une campagne de publicité ou ce qui se passe derrière les murs d'une agence, par Claude Cossette et René Déry. (Yves Tessier)

34 (1988), no 1, p. $39-40$
Des actions culturelles pour aujourd'hui : programme d'action du ministère des Affaires culturelles. (Gabriel Dussault)

30 (1984), no 2, p. 64-67

Des bibliothèques pour tous : lignes directrices pour les services de bibliothèque offerts aux canadiens handicapés. (Sophie Janik)

37 (1991), no 1, p. $42-43$

Des livres et des jeunes (vol.1, no 1-). (Jean-Pierre Chabot)

26 (1980), no 2, p. 119-122

(Descamps, Bernard). Association française des documentalistes et bibliothécaires spécialisés. Manuel du bibliothécaire documentaliste travaillant dans les pays en développement

24 (1978), no 4, p. $209-210$

(Deschâtelets, Gilles). Association française des documentalistes et des bibliothécaires spécialisés. Édition électronique et documentation... du plomb à l'électron

31 (1985), no 4, p. 170-171

(Deschâtelets, Gilles). Coulaud, Philippe. La télématique documentaire à l'épreuve. Usages et usagers des bases de données à la BPI

$$
30 \text { (1984), no 1, p. } 32
$$

Deschâtelets, Gilles. Enquête sur les services de téléréférence au Canada. (Jerry Bull)

27 (1981), no 2, p. 83-84

Deschênes, Gaston. Livres blancs et livres verts au Québec, 1974-1981. (Michel Gélinas)

28 (1982), no 3, p. 131-132

Desjardins, Louis. Les services en langue française dans les bibliothèques publiques de l'Ontario par Louis Desjardins et Evelyn Gagné. (Jean-Guy Desroches)

26 (1980), no 4, p. 222-224

Desjardins, Marc. Bibliographie de la Gaspésie. (Jean-Rémi Brault) 35 (1989), no 1, p. 31

Deslauriers, Pierre. Règles de catalogage anglo-américaines (...) sous la direction de Paule Rolland-Thomas avec la collaboration de Pierre Deslauriers. Traduction de Rita Bélanger. (Yvon Richer) 19 (1973), no 4, p. 177-178 
(Desmarais, Huguette). Bethery, Annie. Les périodiques, par Annie Bethery et Jacqueline Gascuel. Avec la collaboration de Michèle Ganot et al.

25 (1979), no 3, p. 167-169

(Desrochers, Monique). Québec (Province). Ministère des Communications. Direction générale de l'édition. Centre de documentation. Répertoire analytique des publications gouvernementales

23 (1977), no 3, p. 170-171

(Desrochers, Monique). Université d'Ottawa. Centre de recherche en civilisation canadienne-française. Le théâtre canadienfrançais; évolution, témoignages, bibliographie

24 (1978), no 3, p. 143-145

(Desroches, Jean-Guy). Desjardins, Louis. Les services en langue française dans les bibliothèques publiques de l'Ontario par Louis Desjardins et Evelyn Gagné

26 (1980), no 4, p. 222-224

(Desroches, Jean-Guy). Discrimination du gouvernement à l'égard des bibliothécaires. Rapport du comité sur le statut et le traitement salarial des bibliothécaires de cégeps

$$
21 \text { (1975), no 1, p. } 57-58
$$

(Desroches, Jean-Guy). Projet progrès : étude sur les bibliothèques publiques canadiennes

28 (1982), no 1 , p. $36-38$

Le développement des systèmes et des services de bibliothèques publiques. Guide pour l'organisation des systèmes nationaux et régionaux de bibliothèques publiques en tant que composantes de la planification générale des services nationaux d'information

voir Campbell, Henry L.

Dewey, Melvil. Abridged Dewey Decimal Classification and Relative Index. Ed.12, edited by John P. Comaroni et al. (Paule Rolland-Thomas)

37 (1991), no 2, p. $82-83$

Dewey, Melvil. Classification décimale de Dewey et index, établis par Melvil Dewey. (Jean-Marc Turgeon et André Paul)

21 (1975), no 1, p. 60-62

Dictionaire de littérature française contemporaine

voir Bonnefoy, Claude
Dictionary of Archival Terminology $=$ Dictionnaire de terminologie archivistique; English and French with Equivalents in Dutch, German, Italian, Russian and Spanish voir Walne, Peter

Dictionnaire alphabétique et analogique de la langue française, $2 e$ éd.

voir Robert, Paul

Dictionnaire anglais-français de l'électronique et de l'électrotechnique voir Renyi, Pierre

Dictionnaire bibliographique du Canada français

voir Barbeau, Victor

Dictionnaire canadien des noms propres voir Veyron, Michel

Dictionnaire de terminologie archivistique voir Walne, Peter

Dictionnaire des anglicismes

voir Colpron, Gilles

Dictionnaire des difficultés de la langue française au Canada

voir Dagenais, Gérard

Dictionnaire des littératures de langue française

voir Beaumarchais, Jean-Pierre de

Dictionnaire des oeuvres littéraires du Québec, sous la direction de Maurice Lemire. (Claude Fournier)

$$
36 \text { (1990), no 1, p. } 35-36
$$

Dictionnaire des oeuvres littéraires du Québec. Tome 1 : des origines à 1900 voir Lemire, Maurice

Dictionnaire du bon français voir Girodet, Jean

Dictionnaire du cinéma québécois voir Houle, Michel

Le dictionnaire du cinéma québécois, sous la direction de Michel Coulombe et Marcel Jean. (Jacques Demers) 35 (1989), no 2, p. $66-67$

Dictionnaire général de la francophonie voir Luthi, J.-J.

Dictionnaire nord-américain de la langue française voir Bélisle, Louis-Alexandre
Dictionnaire pratique des auteurs québécois

voir Hamel, Réginald

Dictionnaire thématique visuel voir Corbeil, Jean-Claude

Dionne, René. Bibliographie de la critique de la littérature québécoise et canadiennefrançaise dans les revues canadiennes (1974-1978), par René Dionne et Pierre Cantin. (Claude Fournier)

37 (1991), no 2, p. 83-84

Directives pour la conception et l'organisation de médiathèques scolaires

voir Carroll, Frances Laverne

Discrimination du gouvernement à l'égard des bibliothécaires. Rapport du comité sur le statut et le traitement salarial des bibliothécaires de cégeps. (Jean-Guy Desroches)

21 (1975), no 1 , p. $57-58$

Dix ans d'activité; cinquième rapport au Premier ministre

voir France. Commission de coordination de la documentation administrative

La documentation à caractère international dans les institutions du Québec. Étude préparée par le groupe de travail sur le projet de création au Québec d'un établissement professionnel d'études internationales (PROCEI)

voir ENAP. Centre de documentation

"La documentation", Méta (numéro spécial), v.25, no 1, (mars 1980)

voir Bélanger, Nycole

Documents cartographiques depuis la découverte de l'Amérique jusqu'à 1820 : inventaire sommaire, [compilé par] Pierre Lépine et Josée Berthelette. (Yves Tessier) 33 (1987), no 2, p. 74-76

(Dostaler, Henriette). Bélisle, Alvine. Guide de lecture pour les jeunes 5 à 13 ans

19 (1973), no 3, p. 136-137

Drôles de bibliothèques... : le thème de la bibliothèque dans la littérature et le cinéma voir Chaintreau, Anne-Marie

(Drolet, Gaétan). Aubin, Paul. Bibliographie de l'histoire du Québec et du Canada 1966-1975

28 (1982), no 3, p. $128-130$ 
(Drolet, Gaétan). Canada. Comité des systèmes unifiés de bibliothèque. Groupe de travail sur la mesure de la performance. Mesure de la performance dans les bibliothèques fédérales : un guide. Performance Measurement in Federal Libraries : A Handbook

27 (1981), no 2, p. 84-86

Drouin, Paquin \& associés. Étude sur le commerce du livre au Québec

23 (1977), no 4, p. 218-220

$D S I / C B$, Service de diffusion sélective de l'information de la Centrale des bibliothèques. (Nicole Lavigne)

31 (1985), no 1, p. $39-40$

Du bon usage des bibliographies

voir Reboul, Jacquette

(Du Breuil, Laval). Van Slype, Georges. Conception et gestion des systèmes documentaires

24 (1978), no 4, p. 208-209

Du texte à limage : le livre illustré au Québec

voir Bernier, Silvie

Dubuc, Pierrette. Thésaurus : Enfance inadaptée, par Pierrette Dubuc, Lise Thibodeau-Brunet et Luc-Louis Lecompte. (Jean Bouthillette et Raymond Caron) 27 (1981), no 2, p. 82-83

Dubuc. Robert. Vocabulalre bilingue de la production télévision. (Jacques Vermette)

29 (1983), no 3, p. 124

(Ducharme, Jacques). Archives publiques du Canada. Union List of Manuscripts in Canadian Repositories / Catalogue collectif des manuscrits des archives canadiennes

$$
22 \text { (1976), no 1, p. 47-49 }
$$

Ducharme, Jacques. Guide provisoire des Archives judiciaires du district de Montréal. (Jean-Rémi Brault)

33 (1987), no 3, p. 107-108

(Ducharme, Yves). Drouin, Paquin \& associés. Étude sur le commerce du livre au Québec

23 (1977), no 4, p. $218-220$

(Dufresne, Nicole). Beaudin, François. Archivistique québécoise

22 (1976), no 2, p. 98-99
(Dufresne, Nicole). Hudrisier, Henri. L'iconothèque : documentation audiovisuelle et banque d'images

30 (1984), no 1, p. $29-30$

(Dumouchel, Bernard). Demers, Jacques. Gestion des documents audiovisuels dans les bibliothèques des collèges francophones du Québec

$$
23 \text { (1977), no 4, p. 217-218 }
$$

Dunnigan, Lise. Analyse des stéréotypes masculins et féminins dans les manuels scolaires au Québec. (Marielle Durand)

22 (1976), no 3, p. 147-149

Durance, Cynthia J. Vers l'établissement d'un réseau de communication bibliographique au Canada. (Maurice Auger)

$$
29 \text { (1983), no 2, p. 77-78 }
$$

(Durand, Marielle). Dunnigan, Lise. Analyse des stéréotypes masculins et féminins dans les manuels scolaires au Québec

22 (1976), no 3, p. 147-149

(Durand, Marielle). Mareuil, André. Le livre et la construction de la personnalité de l'enfant

24 (1978), no 3, p. 152-154

(Durand, Marielle). Szpakowska, JaninaKlara. Ces lectures sauvages; panoramique sur le service de bibliothèque aux jeunes adultes

19 (1973), no 2, p. 89-91

Durand, Marion. L'image dans le livre pour enfants, par Marion Durand et Gérard Bertrand. (Louisette Bergeron-Choquette)

23 (1977), no 1, p. $48-51$

(Dussault, Gabriel). Rapport du Comité d'étude de la politique culturelle fédérale

30 (1984), no 2, p. 64-67

(Dussault, Gabriel). Des actions culturelles pour aujourd'hui : programme d'action du ministère des Affaires culturelles

30 (1984), no 2, p. $64-67$

(Dussault, Gabriel). Rapport de la consultation du ministre des Affaires culturelles du Québec

30 (1984), no 2, p. 64-67
$E$

Eco, Umberto. De bibliotheca. (Jean-Rémi Brault)

32 (1986), no 4, p. 157

Écrits sur l'avenir

voir Allard, Yvon

Écrits sur le cinéma : bibliographie québécoise 1911-1981

voir Fournier-Renaud, Madeleine

L'édition au Québec de 1960 à 1977

voir Cau, Ignace

L'édition du livre populaire : études sur les Éditions Édouard Garand, de l'Étoile, Marquis, Granger Frères, rassemblées et présentées par Jacques Michon. (JeanRémi Brault)

34 (1988), no 4, p. 159

Édition électronique et documentation... du plomb à l'électron

voir Association française des documentalistes et des bibliothécaires spécialisés

Les Éditions du Jour. Une génération d'écrivains

voir Janelle Claude

Édouard-Raymond Fabre, libraire et patriote canadien, 1799-1854

voir Roy, Jean-Louis

ENAP. Centre de documentation. La documentation à caractère international dans les institutions du Québec. Étude préparée par le groupe de travail sur le projet de création au Québec d'un établissement professionnel d'études internationales (PROCEI). (Françoise Sorieul)

30 (1984), no 1, p. 27-28

Encyclopédie de la musique au Canada voir Kallmann, Helmut

L'enfant et les images de la littérature enfantine

voir Danset-Léger, Jacqueline

L'enfant, l'image et le récit voir Escarpit, Denise

Enquête sur les services de téléréférence au Canada

voir Deschâtelets, Gilles

L'enseignement du français au primaire. (François-R. Soucy)

27 (1981), no 4, p. 161-164 
Escarpit, Denise. L'enfant, l'image et le récit. Ouvrage collectif sous la direction de Denise Escarpit. (Louisette BergeronChoquette et Claude Bouchard))

25 (1979), no 3, p. 169-171

Escarpit, Robert. Le livre français : hier, aujourd'hui, demain. Ouvrage collectif sous la direction de Julien Cain, Robert Escarpit, Henri-Jean Martin. (Jean-Louis Roy) 20 (1974), no 1, p. 44-45

États-Unis. Library of Congress. Subject Cataloging Division. Introduction à la huitième édition de Library of Congress Subject Headings. (Monique Arnoldi)

26 (1980), no 4, p. 220-221

Étude sur le commerce du livre au Québec voir Drouin, Paquin \& associés

L'Europe de l'information voir Pelou Pierre

L'évaluation de l'efficacité de la bibliothèque de collège : une approche systémique

voir Groupe de travail sur l'évaluation des bibliothèques de collèges

L'évaluation des bibliothèques universitaires

voir Clavel, Jean-Pierre

L'évolution du rôle social de l'imprimé et de ses agents au Québec. Texte intégral des conférences présentées lors de la série 1979-1980. (Claude Galarneau)

27 (1981), no 4, p. 157-158

L'expertise québécoise en gestion des documents administratifs; bibliographie thématique et chronologique 1962-1987 voir Roberge, Michel

$\mathbf{F}$

Fabre, Jean-Bernard. Le Québec mis en chiffres : statistiques sociales et économiques sur les régions et les villes du Québec. (Roger de la Sablonnière)

35 (1989), no 3, p. 121-123

Faubert, Michel. Recueil d'exemples itlustrant les Règles de catalogage angloaméricaines. Édition provisoire sous la direction de Lise Calvé-Woodrough et Michel Faubert. Avec la collaboration de Louise Gagnon (et autres). (Léonard Nadeau)

21 (1975), no 4, p. 231-232
(Faure, Sylvie). Québec. Bibliothèque nationale du Québec. Statistiques de l'édition aú Québec en 1990

38 (1992), no 4, p. 214-215

Faurie, Albertine. La pédagogie de la documentation dans le contexte de l'audiovisuel et des mass-média. (Jean de Bonville)

27 (1981), no 2, p. 86-87

La femme et la société québécoise voir Houle, Ghislaine

Figuier, Richard dirigé par. La bibliothèque, miroir de l'âme, mémoire du monde. (Jean-Rémi Brault)

38 (1992), no 4, p. 216-217

Filion, Léo. L'information électronique au Québec : guide pratique des services d'information en ligne, par Louis Bellemare et Léo Filion. (Yves Tessier)

33 (1987), no 4, p. 137-138

(Filion, Louise). Proceedings of the $\mathrm{Na}$ tional Bibliographies Seminar, hold under the auspices of the IFLA Division of Bibliographic Control

36 (1990), no 4, p. 142-143

Filion, Louise. Classification des publications gouvernementales du Québec. $2 e$ éd. (Paule Rolland-Thomas)

21 (1975), no 1, p. 58-60

Films et vidéocassettes - Catalogue 1986. 4 e éd.

voir CEGEP de Trois-Rivières

Films et vidéos documentaires pour enfants. (Louis Frémont) 35 (1989), no 4, p. 191-192

Fleury Mesplet (1734-1794); diffuseur des lumières au Québec

voir Lagrave, Jean-Paul de

Fontaine, France. Principes directeurs pour la rédaction d'objectifs d'apprentissage en bibliothéconomie, en sciences de l'information et en archivistique. (Françoise Burton)

35 (1989), no 4, p. 190-191

(Fontaine, Marcel). Rivet-Panaccio, Colette. Les bibliothèques canadiennes à l'ère de l'automatisation : synthèse bibliographique 1970-72, par Colette RivetPanaccio, Amal Awad et Robert Cardinal 19 (1973), no 2, p. 93-94
La formation des bibliothécaires et le développement des bibliothèques universitaires en Afrique

voir Bousso, Amadou

Fortier, André. Dictionnaire bibliographique du Canada français, par Victor Barbeau et André Fortier. (Yvan Lamonde)

21 (1975), no 3, p. 172-173

(Fortier, Jean-Louis). Canada. Statistique Canada. Division de l'éducation, des sciences et de la culture. Section des beaux-arts et des médias. Literary and Journalistic Awards in Canada / Les prix de littérature et de journalisme au Canada, 1923-1973

23 (1977), no 2, p. 117-120

Fortier, Marie-France. Index du Bulletin des recherches historiques. (Maurice Pellerin)

35 (1989), no 1, p. 28

(Fortin Jean-Luc). Bousso, Amadou. La formation des bibliothécaires et le développement des bibliothèques universitaires en Afrique

29 (1983), no 2, p. $80-82$

(Fortin, Jean-Luc). Indicateurs d'activités culturelles au Québec, édition 1990

37 (1991), no 3, p. 123-124

(Fortin, Jean-Luc). Point de repère : index analytique d'articles de périodiques québécois et étrangers

31 (1985), no 1, p. $41-43$

(Fournier Claude). Lemire, Maurice et al. La vie littéraire au Québec

37 (1991), no 4, p. 163-164

(Fournier, Claude). Bérubé, Robert-Claude. Une sélection de 100 films tirés de romans en vidéocassettes

38 (1992), no 4, p. 215

(Fournier, Claude). Chantigny, Louis et al. Bernard Valiquette

32 (1986), no 1-2, p. 56-57

(Fournier, Claude). Dictionnaire des oeuvres littéraires du Québec, sous la direction de Maurice Lemire

36 (1990), no 1, p. $35-36$

(Fournier, Claude). Dionne, René. Bibliographie de la, critique de la littérature québécoise et canadienne-française dans les revues canadiennes (1974-1978), par René Dionne et Pierre Cantin

37 (1991), no 2, p. 83-84 
(Fournier, Claude). Renyi, Pierre. Dictionnaire anglais-français de l'électronique et de l'électrotechnique, par Pierre Renyi et Dominique Amrouni

$$
33 \text { (1987), no 1, p. } 29-30
$$

(Fournier, Claude). Robitaille, Denis. Theses in Canada: A Bibliographic Guide = Thèses au Canada : guide bibliographique. $2 \mathrm{e}$ édition

34 (1988), no 1, p. $37-38$

(Fournier, Sylvie). Savard, Réjean. Principes directeurs pour l'enseignement du marketing dans la formation des bibliothécaires, documentalistes et archivistes

35 (1989), no 3, p. 124

Fournier-Renaud, Madeleine. Écrits sur le cinéma : bibliographie québécoise 19111981, par Madeleine Fournier-Renaud et Pierre Véronneau. (Régis Mathieu)

30 (1984), no 3, p. 103-104

France-actualité, index de la presse écrite française. (Jerry Bull)

$$
25 \text { (1979), no 3, p. 162-164 }
$$

France. Commission de coordination de la documentation administrative. Dix ans d'activité; cinquième rapport au Premier ministre. (Gaston Bernier)

29 (1983), no 3, p. $119-120$

Francophonie édition, no 1- nov. 1972(Jacques Paradis)

19 (1973), no 3, p. 134-136

Franklin, Karen. Un titre s'il vous plait : conseils à l'intention des éditeurs de revues spécialisées, rapports, journaux et autres publications en série canadiennes. (Nicole Lavigne)

31 (1985), no 2, p. 73-74

(Frémont, Louis). Films et vidéos documentaires pour enfants

35 (1989), no 4, p. 191-192

La fréquentation des bibliothèques et des librairies au Canada

voir Ben-Gera, Michel

Frigon, Claire Renaud

voir Renaud-Frigon, Claire
G

(Gagné, Evelyn). Les services en langue française dans les bibliothèques publiques de l'Ontario par Louis Desjardins et Evelyn Gagné. (Jean-Guy Desroches)

26 (1980), no 4, p. 222-224

Gagné, Marc. Gilles Vigneault : bibliographie descriptive et critique, discographie, filmographie, iconographie, chronologie. (Normand Cormier)

24 (1978), no 4, p. 203-205

Gagné, Raymond. Publications parlementaires québécoises : catalogue collectif. (Gaston Bernier)

$$
32 \text { (1986), no } 1-2 \text {, p. } 60-61
$$

(Gagnon, Gilbert). La fréquentation des bibliothèques et des librairies au Canada, par Michel Ben-Gera, Peat Warwick and Partners et Brian L. Kingsley

$$
27 \text { (1981), no 4, p. 164-166 }
$$

(Gagnon, Gilbert). Seibel, Bernadette. Au nom du livre; analyse sociale d'une profession : les bibliothécaires

35 (1989), no 2, p. $62-64$

(Gagnon, Gilbert). Sociologie du livre et de la lecture

$$
25 \text { (1979), no 4, p. 221-225 }
$$

(Gagnon, Gilbert). Statistiques culturelles du Québec 1971-1982

$$
32 \text { (1986), no 3, p. 100-101 }
$$

Gagnon, Louise. Recueil d'exemples itlustrant les Règles de catalogage angloaméricaines. Édition provisoire sous la direction de Lise Calvé-Woodrough et Michel Faubert. Avec la collaboration de Louise Gagnon (et autres). (Léonard Nadeau)

$$
21 \text { (1975), no 4, p. 231-232 }
$$

(Gagnon-Arguin, Louise). Walne, Peter et al. Dictionary of Archival Terminology = Dictionnaire de terminologie archivistique; English and French with Equivalents in Dutch, German, Italian, Russian and Spanish.

$$
32 \text { (1986), no 3, p. 101-102 }
$$

Gagnon-Arguin, Louise. Une introduction au contrôle d'autorité pour le traitement des noms propres en archivistique. (Jean-Yves Rousseau)

37 (1991), no 3, p. 124-125
(Galarneau, Claude). L'évolution du rôle social de l'imprimé et de ses agents au Québec. Texte intégral des conférences présentées lors de la série 1979-1980

27 (1981), no 4, p. 157-158

(Galarneau, Pierrette). Vinet, Bernard. Bibliographie sur les personnes âgées: répertoire des livres et des périodiques de la collection de la Bibliothèque de I'Université Laval, par Bernard Vinet et Louis-Philippe Jolicoeur

24 (1978), no 3, p. 151-152

(Gallichan, Gilles). Aubin, Paul. Bibliographie de l'histoire du Québec et du Canada 1966-1975

28 (1982), no 3, p. $128-130$

(Gallichan, Gilles). Brodeur, Raymond et al. Les catéchismes au Québec, 17021963

37 (1991), no 3, p. 122-123

(Gallichan, Gilles). Lajeunesse, Marcel. Les Sulpiciens et la vie culturelle à Montréal au XIXe siècle

29 (1983), no 2, p. 78-79

(Gallichan, Gilles). Lamonde, Yvan. Gens de parole. Conférences publiques, essais et débats à l'Institut canadien de Montréal 1845-1871

37 (1991), no 4, p. 165

(Gallichan, Gilles). Lamonde, Yvan. Les bibliothèques de collectivités à Montréal (17e-19e siècle) : sources et problèmes

26 (1980), no 2, p. 118-119

(Gallichan, Gilles). Lamonde. Yvan. Les bibliothèques personnelles au Québec : inventaire analytique et préliminaire des sources, par Yvan Lamonde et Daniel Olivier

29 (1983), no 4, p. 167

(Gallichan, Gilles). Luthi, J.J. Dictionnaire général de la francophonie, par J.-J. Luthi, A. Viatte, G. Zanarini

34 (1988), no 2, p. $79-80$

(Gallichan, Gilles). Québec. Archives nationales. Guide. Archives écrites d'origine privée conservées au Centre d'archives de Québec et de ChaudièreAppalaches

38 (1992), no 3, p. 174-175 
(Gallichan, Gilles). Thériault, Michel. Le livre religieux au Québec depuis les débuts de l'imprimerie jusqu'à la Confédération (1764-1867) : relevé statistique et essai d'interprétation

$$
24 \text { (1978), no 1, p. } 48-49
$$

(Gallichan, Gilles). Vlach, Milada. Catalogue collectif des impressions québécoises. 1764-1820, par Milada Vlach et Yolande Buono

30 (1984), no 4, p. $142-143$

Gallichan, Gilles. Livre et politique au BasCanada, 1791-1849. (Marcel Lajeunesse)

$$
38 \text { (1992), no 3, p. 171-173 }
$$

(Gallichan, Gilles). Lemoine, Roger. Catalogue de la bibliothèque de Louis-Joseph Papineau

29 (1983), no 4, p. 167

Gallouédec-Génuys, Françoise. Une société sans papier. (Jean Goulet)

$$
37 \text { (1991), no 4, p. 165-167 }
$$

Garon, Denise. La classification des jeux et des jouets - Le système ESAR. (Gilles Brougère)

$$
33 \text { (1987), no 1, p. } 30-31
$$

Gascuel, Jacqueline. Les périodiques, par Annie Bethery et Jacqueline Gascuel. Avec la collaboration de Michèle Ganot et al. (Huguette Desmarais)

25 (1979), no 3, p. 167-169

Gastaldy, Suzanne Bertrand

voir Bertrand-Gastaldy, Suzanne

Gaudette, Pierre. La répartition du budget des collections au Service des bibliothèques de I'Université de Sherbrooke. (Claude Busque)

32 (1986), no 3, p. $99-100$

Gauvin, Daniel. Guide canadien du livre rare / Canadian Guide to Rare Books. (Ginette Vadnais)

36 (1990), no 3, p. $109-110$

(Gélinas, Michel). Deschênes, Gaston. Livres blancs et livres verts au Québec, 1964-1981

28 (1982), no 3, p. 131-132

(Gélinas, René). Beauchesne, Yves. Animer la lecture : pour faire lire...; guide pratique

33 (1987), no 1, p. 32
(Genest, Nicole). Classe PS 8000 . Cadre de classification de la littérature canadienne (2e éd.)

25 (1979), no 1 , p. $50-51$

Gens de parole. Conférences publiques, essais et débats à I'Institut canadien de Montréal 1845-1871

voir Lamonde, Yvan

Génuys, Françoise Gallouédec

voir Gallouédec-Génuys, Françoise

A Geo-documentary Profile of the Commonwealth Developing Countries (Gaston Bernier)

$$
38 \text { (1992), no 3, p. 173-174 }
$$

Germanaud, Marie-Claire. La bibliothèque publique en milieu rural et dans les petites agglomérations. (Réal Messier)

29 (1983), no 4, p. 169

Gestion des documents audiovisuels dans les bibliothèques des collèges francophones du Québec voir Demers, Jacques

La gestion des publications officielles (Gaston Bernier)

$$
37 \text { (1991), no 1, p. } 41
$$

La gestion documentaire

voir Raiffaud, Joël

(Giguère, Pierre). Beaumarchais, JeanPierre de. Dictionnaire des littératures de langue française, par Jean-Pierre de Beaumarchais, Daniel Couty et Alain Rey

31 (1985), no 2, p. 74-75

(Giguère, Pierre). Boivin, Aurélien. Le conte littéraire québécois au XIXe siècle : essai de bibliographie critique et analytique 22 (1976), no 3, p. 149-150

(Giguère, Richard). Canada. Statistique Canada. Division de l'éducation, des sciences et de la culture. Section des beaux-arts et des médias. Literary and Journalistic Awards in Canada / Les prix de littérature et de journalisme au Canada, 1923-1973

23 (1977), no 2 , p. $117-120$

Gilles Vigneault : bibliographie descriptive et critique, discographie, filmographie, iconographie, chronologie voir Gagné, Marc

Girodet, Jean. Dictionnaire du bon français. (Louis-Jean Rousseau) 32 (1986), no 1-2, p. 61-62
(Goulet, Jean). Gallouédec-Génuys, Françoise. Une société sans papier 37 (1991), no 4, p. 165-167

Gourd, Benoit-Beaudry. Bibliographie de l'Abitibi-Témiscamingue. (Nicole Côté)

24 (1978), no 1, p. 45-47

Gouvernement du Québec. Rapport de recherche sur les bibliothèques scolaires et l'enseignement élémentaire dans la région de l'Estrie. (André Jacques)

20 (1974), no 2, p. 101-104

(Grolier, Eric de). Québec. Comité d'étude d'un réseau des bibliothèques québécoises pour le traitement en coopération de leur documentation. Vers un réseau québécois des bibliothèques. Rapport du CERBIQ

25 (1979), no 4, p. 217-219

Groupe de travail sur l'évaluation des bibliothèques de collèges. L'évaluation de l'efficacité de la bibliothèque de collège : une approche systémique. (Monique Potin) 26 (1980), no 1, p. 49-51

Groupe de travail sur la mesure de la performance

voir Canada. Comité des systèmes unifiés de bibliothèque. Groupe de travail sur la mesure de la performance

Groupe de travail sur la normalisation du catalogage

voir Canada. Groupe de travail sur la normalisation du catalogage

(Guay, Jacques). Beaulieu, André. La presse québécoise des origines à nos jours, 1920-1934, 1935-1944, par André Beaulieu et Jean Hamelin 31 (1985), no 4, p. 172-173

Guay, Jean-Pierre. Lorsque notre littérature était jeune. Entretiens avec Pierre Tisseyre. (Marcel Lajeunesse)

29 (1983), no 4, p. 166

Guerre, paix et désarmement. Bibliographie thématique en langue française voir Poole, Erik

Guide canadien du livre rare / Canadian Guide to Rare Books

voir Gauvin, Daniel

Guide d'aménagement des bibliothèquescentres documentaires des écoles élémentaires

voir Québec. Ministère de l'Education. Direction générale de l'administration 
Guide de lecture pour les jeunes 5 à 13 ans voir Bélisle, Alvine

Guide de recherche documentaire en matière de publications parlementaires et gouvernementales du Québec

voir Bourgault, Jacques

Guide de recherche documentaire en science politique

voir Laghzali, Mohamed

Guide de redaction bibliographique

voir Thibault, Danielle

Guide des archives littéraires

voir Archives nationales du Canada. Division des manuscrits

Guide des sources d'archives sur le Canada français au Canada

voir Archives publiques du Canada

Guide pour la littérature pour la jeunesse voir Soriano, Marc

Guide pratique d'indexation en vocabulaire libre

voir Breton, Jean

Guide pratique des techniques documentaires

voir Guinchat, Claire

Guide provisoire des Archives judiciaires du district de Montréal

voir Ducharme, Jacques

(Guilloton, Noëlle). Bélisle, LouisAlexandre. Dictionnaire nord-américain de la langue française

28 (1982), no 3, p. $135-136$

(Guilloton, Noëlle). Decroux-Masson, Annie. Papa lit, maman coud; les manuels scolaires en bleu et rose

26 (1980), no 2, p. 123

Guinchat, Claire. Guide pratique des techniques documentaires, par Claire Guinchat et Yolande Skouri, avec la collaboration de Marie-Pierre Alix. (Richard Paré)

37 (1991), no 2, p. 83

Guinchat, Claire. Introduction générale aux sciences et techniques de la documentation par Claire Guinchat et Michel Menou. (Suzanne Bertrand-Gastaldy)

29 (1983), no 1, p. $41-43$
H

Les habitudes de lecture des québécois voir Bergeron, Alain

Les habitudes de lecture des québécois de 10-12 ans

voir Héon, Gérard

Hamel, Réginald. Dictionnaire pratique des auteurs québécois, par Réginald Hamel, John Hare et Paul Wyczynski. (Patrick Coppens)

23 (1977), no 2, p. 109-112

Hamelin, Jean et al. Brochures québécoises 1764-1972. (John E. Hare)

28 (1982), no 4, p. 170-171

Hamelin, Jean. La presse québécoise des origines à nos jours, 1920-1934, 19351944, par André Beaulieu et Jean Hamelin. (Jacques Guay)

31 (1985), no 4, p. 172-173

Hamelin, Jean. La presse québécoise des origines à nos jours. Tome premier : 17641859, par André Beaulieu et Jean Hamelin. (Marcel Lajeunesse)

19 (1973), no 3, p. 138

Handicap, lecture et bibliothèques. (Sophie Janik)

$$
37 \text { (1991), no 1, p. } 43
$$

Hanse, Joseph. Nouveau dictionnaire des difficultés du français moderne. (LouisJean Rousseau)

$$
32 \text { (1986), no 1-2, p. 61-62 }
$$

(Hare, John E.). Hamelin, Jean et al. Brochures québécoises 1764-1972

28 (1982), no 4, p. 170-171

(Hare, John E.). Québec. Bibliothèque nationale du Québec. Laurentiana parus avant 1821, par Milada Vlach, avec la collaboration de Yolande Buono

23 (1977), no 4, p. 215-216

Hare, John. Dictionnaire pratique des auteurs québécois, par Réginald Hamel, John Hare et Paul Wyczynski. (Patrick Coppens)

23 (1977), no 2, p. 109-112

Hébert, Françoise. La photocopie dans les bibliothèques canadiennes. (Jules Larivière)

34 (1988), no 3, p. $111-112$
Henry, Ginette. Répertoire des périodiques québécois: 1re partie, par Ginette Henry. (Colette Rivet-Panaccio)

21 (1975), no 4, p. 227-228

Héon, Gérard. Les habitudes de lecture des québécois de 10-12 ans. (Réal Messier)

29 (1983), no 2, p. $79-80$

Herzhaft, Gérard. Pour une médiathèque : guide de l'audiovisuel à l'usage des candidats aux concours et examens des bibliothèques. (Silvie Delorme)

29 (1983), no 3, p. 120-121

Histoire des bibliothèques françaises. (Marcel Lajeunesse)

38 (1992), no 4, p. $210-212$

L'histoire des idées au Québec, 17601960 voir Lamonde, Yvan

Hoa-Thuy, Pham-Dang. L'utilisation des moyens didactiques imprimés et audiovisuels par les professeurs au secondaire: une étude préliminaire par Marcel Bélanger et Hoa-Thuy Pham-Dang. (Georgette Lapointe et Rosaire Pelletier)

23 (1977), no 3, p. 171-173

Hoa-Thuy, Pham-Dang. Les centres de ressources éducatives dans l'enseignement secondaire : exploration de la diversité des modèles, des situations et des problèmes locaux par Christian Bergeron et Hoa-Thuy Pham-Dang. (Georgette Lapointe et Rosaire Pelletier)

23 (1977), no 3, p. 171-173

(Hogue, Gisèle). Bibliothèque nationale du Québec. Catalogue des manuscrits, dressé par le département des manuscrits

25 (1979), no 2, p. 105-107

Houde, Denis. Index rétrospectif, 19551979. (Roland Auger)

30 (1984), no 1, p. 35

Houle, Ghislaine. La femme et la société québécoise. (Hélène Larose et Andrée Martin)

21 (1975), no 3, p. 169-171

Houle, Michel. Dictionnaire du cinéma québécois, par Michel Houle et Alain Julien. (Pierre Véronneau)

25 (1979), no 3, p. 165-167

(Houyoux, Philippe). Tega, Vasile. Information et documentation, facteurs de progrès dans l'entreprise

22 (1976), no 2, p. $97-98$ 
Houyoux, Philippe. Bibliographie de I'histoire de l'éducation au Québec des origines à 1960, compilée par Philippe Houyoux. (François Rheault)

$$
25 \text { (1979), no 1, p. } 56
$$

(Hudon, Michèle). Théorie et pratique dans l'enseignement des sciences de l'information. Bridging the Gap between Theory and Practice. Textes colligés par Réjean Savard

35 (1989), no 2, p. 61-62

(Hudon, Michèle). Union des écrivains québécois. Petit dictionnaire des écrivains

26 (1980), no 1, p. 55-56

Hudrisier, Henri. L'iconothèque; documentation audiovisuelle et banque d'images. (Nicole Dufresne)

30 (1984), no 1, p. $29-30$

Humanisme et bibliothèques : essai sur la philosophie de la bibliothéconomie voir Cossette, André

Hurtubise, Rolland. Informatique et information; la conception des systèmes d'information; les banques de données. (Claude Bonnelly)

25 (1979), no 2, p. 103-105

Huvelin, France Latreille

voir Latreille-Huvelin, France

I

L'iconothèque; documentation audiovisuelle et banque d'images

voir Hudrisier, Henri

La Sablonnière, Roger de

voir Sablonnière, Roger de la

Lacroix, Jean-Michel. Anatomie de la presse ethnique au Canada. (André Beaulieu)

36 (1990), no 1, p. $36-37$

Les idéologies au Québec

voir Monière, Denis

ILO

voir Bureau international du travail

ILO Thesausus - Thésaurus B.I.T. - Tesauro O.I.T.

voir Bureau international du travail

L'image dans le livre pour enfants voir Durand, Marion
Index analytique du journal Le Monde diplomatique, 1954-1983. (Gaston Bernier)

30 (1984), no 4, p. 141-142

Index anglais-français des termes utilisés dans le Répertoire de vedettes-matière voir Université Laval. Bibliothèque. Service de catalogage

Index de l'actualité vue à travers la presse écrite. (Jerry Bull)

23 (1977), no 2, p. 114-116

Index des lois à caractère privé du Québec, $1867-1975$

voir Québec. Bibliothèque de la législature

Index du Bulletin des recherches historiques

voir Fortier, Marie-France

Index du fichier de terminologie. (André Lapierre)

19 (1973), no 3, p. 139-141

Index rétrospectif, 1955-1979

voir Houde, Denis

Index sur la guerre et la paix : index relationnel de mots-clés pour SONJA, banque de données informatisées sur la guerre, la paix et le désarmement en langue française

voir Bourret Annie

Indicateurs d'activités culturelles au Québec, édition 1990. (Jean-Luc Fortin)

37 (1991), no 3, p. 123-124

L'indispensable en documentation; les outils de travail

voir Bourget, Manon

Info-Carto 02 : Répertoire cartobibliographique sur la région 02 et Moyen Nord, par Guy Biron et France Gagnon sous la direction de Richard Bouchard. (Yves Tessier)

33 (1987), no 2, p. 74-76

Infodex: index de la Presse. (Jules Lord)

33 (1987), no 1, p. $32-34$

L'information électronique au Québec : guide pratique des services d'information en ligne

voir Bellemare, Louis
Information et documentation : attente des pays en voie de développement; Information and Documentation: The Expectations of Developing Countries. Actes du Colloque organisé par le comité des relations internationales de I'ASTED... Montréal, 16-17 novembre 1981. (JeanPaul Plante)

29 (1983), no 3, p. 121-122

Information et documentation, facteurs de progrès dans l'entreprise

voir Tega, Vasile

Information et liberté. Rapport de la Commission d'étude sur l'accès du citoyen à l'information gouvernementale et sur la protection des renseignements personnels. (Daniel Allaire)

27 (1981), no 4, p. 159-161

Information et pédagogie

voir Reboul, Elie

L'information gouvernementale au Québec : bibliographie analytique

voir Brière, Jean-Marie

Informatique et information; la conception des systèmes d'information; les banques de données

voir Hurtubise, Rolland

Initiation à la bibliographie scientifique voir Such, Marie-France

Les instituts de vie consacrée au Canada depuis les débuts de la Nouvelle-France jusqu'à aujourd'hui; notes historiques et références. The Institutes of Consacred Life in Canada from the Beginning of the New France up to the Present; Historical Notes and References

voir Thériault, Michel

L'intelligence artificielle : promesse et réalités

voir Bonnet, Alain

L'inter Atlas : les ressources du Québec et du Canada

voir Centre d'études en enseignement au Canada

Introduction à la huitième édition de Library of Congress Subject Headings

voir Etats-Unis. Library of Congress. Subject Cataloging Division

Introduction aux ouvrages généraux de référence sur la communication et les mass media

voir Bonville, Jean de 
Introduction générale aux sciences et techniques de l'information et de la documentation

voir Guinchat, Claire

Inventaire général des bibliographies nationales rétrospectives $=$ Retrospective National Bibliographies : An International Directory

voir Beaudiquez, Marcelle

Isnard, F. Lalande

voir Lalande-lsnard, F.

$J$

(Jacques, André). Gouvernement du Québec. Rapport de recherche sur les bibliothèques scolaires et l'enseignement élémentaire dans la région de l'Estrie

20 (1974), no 2, p. 101-104

Janelle, Claude. Les Éditions du Jour. Une génération d'écrivains. (Marcel Lajeunesse)

29 (1983), no 4, p. 166

(Janik, Sophie). Bonnet, Alain. L'intelligence artificielle : promesse et réalités

31 (1985), no 4, p. 171-172

(Janik, Sophie). Des bibliothèques pour tous : lignes directrices pour les services de bibliothèque offerts aux canadiens handicapés

37 (1991), no 1, p. $42-43$

(Janik, Sophie). Handicap, lecture et bibliothèques

37 (1991), no 1, p. 43

Janik, Sophie. Bibliographie québécoise sur les personnes handicapées, établie par Sophie Janik, avec la collaboration de Edward A. Collister et Lise Brunet. (JeanRémi Brault)

35 (1989), no 1, p. $29-30$

Janik, Sophie. Thésaurus : délinquance juvénile, par Vesna Blazina et Sophie Janik. (Lise Brunet)

28 (1982), no 1, p. $35-36$

Jeunes gens : thésaurus; essai de systématisation des connaissances spécifiques à la classe d'âge 13-25 ans

voir Szpakowska, Janina-Klara
Jolicoeur, Louis-Philippe. Bibliographie sur les personnes âgées : répertoire des livres et des périodiques de la collection de la Bibliothèque de l'Université Laval, par Bernard Vinet et Louis-Philippe Jolicoeur. (Pierrette Galarneau)

$$
24 \text { (1978), no 3, p. 151-152 }
$$

Jolicoeur, Louis-Philippe. Revues d'analyse et d'indexation. Répertoire annoté des revues d'analyse et d'indexation de la collection de la Bibliothèque de l'Université Laval. (Denis Boucher)

23 (1977), no 3, p. 174-175

(Jonynas, V. Alexandre). Conférence nationale sur l'état de la bibliographie au Canada, Vancouver, les 22,23 et 24 mai 1974. Comptes rendus

24 (1978), no 2, p. $92-95$

(Jonynas, V. Alexandre). Québec. Bibliothèque nationale. Les ouvrages de référence du Québec. Supplément 1967-1974

22 (1976), no 2, p. 91-93

Journal des débats. Index 1988/1989

voir Québec. Assemblée nationale

(Julien, Alain). Les cinémas canadiens. Dossier établi sous la direction de Pierre Véronneau

25 (1979), no 3, p. 164-165

Julien, Alain. Dictionnaire du cinéma québécois, par Michel Houle et Alain Julien. (Pierre Véronneau)

25 (1979), no 3, p. 165-167

Jutras, Pierre, sous la dir. de. Annuaire du cinéma québécois 1990. (Jean-Rémi Brault)

38 (1992), no 4, p. 215-216

K

Kallmann, Helmut et al. Encyclopédie de la musique au Canada. (Michelle Bachand)

30 (1984), no 2, p. 61-62

Kingsley, Brian L. La fréquentation des bibliothèques et des librairies au Canada, par Michel Ben-Gera, Peat Warwick and Partners et Brian L. Kingsley. (Gilbert Gagnon)

27 (1981), no 4, p. 164-166
Laflèche, Michelle Bélisle

voir Bélisle-Laflèche, Michelle

Lafleur, Raymonde Couture

voir Couture-Lafleur, Raymonde

Lafortune, Hélène et al. Parchemin s'explique...; guide de dépouillement des actes notariés du Québec ancien. (JeanRémi Brault)

35 (1989), no 3, p. 126

Laghzali, Mohamed. Guide de recherche documentaire en science politique, par Mohamed Laghzali et Evelyne Tardif. (Gaston Bernier)

34 (1988), no 2 , p. $80-81$

Lagrave, Jean-Paul de. Fleury Mesplet (1734-1794); diffuseur des lumières au Québec. (Jean-Rémi Brault)

32 (1986), no 1-2, p. 55-56

(Lajeunesse, Marcel). Beaulieu, André. La presse québécoise des origines à nos jours. Tome premier : 1764-1859, par André Beaulieu et Jean Hamelin

19 (1973), no 3, p. 138

(Lajeunesse, Marcel). Campbell, Henry L. Le développement des systèmes et des services de bibliothèques publiques. Guide pour l'organisation des systèmes nationaux et régionaux de bibliothèques publiques en tant que composante de la planification générale des services nationaux d'information

31 (1985), no 2, p. 75-76

(Lajeunesse, Marcel). Cau, Ignace. L'édition au Québec de 1960 à 1977

28 (1982), no 3 , p. 127-128

(Lajeunesse, Marcel). Classe FC. Cadre de classification de l'histoire du Canada

23 (1977), no 2, p. 112-114

(Lajeunesse, Marcel). Gallichan, Gilles. Livre et politique au Bas-Canada, 17911849

38 (1992), no 3, p. 171-173

(Lajeunesse, Marcel). Guay, Jean-Pierre. Lorsque notre littérature était jeune. Entretiens avec Pierre Tisseyre

29 (1983), no 4, p. 166

(Lajeunesse, Marcel). Histoire des bibliothèques françaises

38 (1992), no 4, p. 210-212 
(Lajeunesse, Marcel). Janelle, Claude. Les Éditions du Jour. Une génération d'écrivains

$$
29 \text { (1983), no 4, p. } 166
$$

(Lajeunesse, Marcel). Lamonde, Yvan. L'histoire des idées au Québec, 1760-1960

37 (1991), no 3, p. 124

(Lajeunesse, Marcel). Revai, Elisabeth. Alexandre Vattemare trait d'union entre deux mondes

21 (1975), no 4, p. 228-229

(Lajeunesse, Marcel). Richter, Noë. Les bibliothèques populaires

$$
26 \text { (1980), no 1, p. 56-58 }
$$

Lajeunesse, Marcel. Les bibliothèques des collèges d'enseignement général et professionnel du Québec : étude de leur évolution, (1969-1983). (Jean-Rémi Brault)

32 (1986), no 1-2, p. 54-55

Lajeunesse, Marcel. Les Sulpiciens et la vie culturelle à Montréal au XIXe siècle. (Gilles Gallichan)

29 (1983), no 2, p. 78-79

Lajeunesse, Marcel. Répertoire des écoles des sciences de l'information. (Abdelaziz Abid)

26 (1980), no 4, p. $225-227$

Lalande-lsnard, F. Les bibliothèques dans trois pays de l'ouest africain (Guinée, Mali, Sierra Leone). (Jean de Chantal)

$$
22 \text { (1976), no 4, p. 199-203 }
$$

(Laliberté, Madeleine). Blais, Raymond. Vocabulaire libre en indexation, par Raymond Blais et Yves Courrier

$$
25 \text { (1979), no 2, p. 107-108 }
$$

(Lamonde, Yvan). Barbeau, Victor. Dictionnaire bibliographique du Canada français, par Victor Barbeau et André Fortier

$$
21 \text { (1975), no 3, p. 172-173 }
$$

Lamonde, Yvan. Gens de parole. Conférences publiques, essais et débats à I'Institut canadien de Montréal, 1845-1871. (Gilles Gallichan)

37 (1991), no 4, p. 165

Lamonde, Yvan. L'histoire des idées au Québec, 1760-1960. (Marcel Lajeunesse)

37 (1991), no 3, p. 124

Lamonde, Yvan. La librairie et l'édition à Montréal, 1776-1920. (Jacques Cotnam) 38 (1992), no 4, p. 212-213
Lamonde, Yvan. Les bibliothèques de collectivités à Montréal (17e-19e siècle) : sources et problèmes. (Gilles Gallichan) 26 (1980), no 2, p. 118-119

Lamonde, Yvan. Les bibliothèques personnelles au Québec : inventaire analytique et préliminaire des sources, par Yvan Lamonde et Daniel Olivier. (Gilles Gallichan)

$$
29 \text { (1983), no 4, p. } 167
$$

Lamothe, Madeleine. Archives des femmes du Québec; guide sommaire. Assistée de Ghislaine Fecteau et Pierrette Lalancette. (Jean-Rémi Brault)

$$
37 \text { (1991), no 2, p. 85-86 }
$$

Lamy-Rousseau, Françoise. Traitement automatisé des documents multimedia avec les systèmes ISBD unifié, LamyRousseau et PRECIS. Propositions SILP. (Louise MacKay et Conrad Corriveau) 21 (1975), no 4, p. 229-231

Lamy-Rousseau, Françoise. Uniformisation des règles de catalogage des documents visuels et sonores: description d'une expérience, par Françoise LamyRousseau. En collab. avec Martine Paquet. (Pierre Manseau)

$$
20 \text { (1974), no } 1 \text {, p. 50-51 }
$$

Langlois, Simon et al. La société québécoise en tendances 1960-1990. (Roger de la Sablonnière)

38 (1992), no 4, p. 213-214

(Lapierre, André). Index du fichier de terminologie

$$
19 \text { (1973), no } 3 \text {, p. } 139-141
$$

(Lapointe, Georgette). Bélanger, Marcel. L'utilisation des moyens didactiques imprimés et audiovisuels par les professeurs au secondaire : une étude préliminaire, par Marcel Bélanger et Hoa-Thuy Pham-Dang

$$
23 \text { (1977), no 3, p. 171-173 }
$$

(Lapointe, Georgette). Bergeron, Christian. Les centres de ressources éducatives dans l'enseignement secondaire : exploration de la diversité des modèles, des situations et des problèmes locaux, par Christian Bergeron et Hoa-Thuy Pham-Dang

$$
23 \text { (1977), no 3, p. 171-173 }
$$

(Lapointe, Georgette). Reboul, Elie. Information et pédagogie

24 (1978), no 4, p. 211-212
(Lapointe, Georgette). Szpakowska, Janina-Klara. Profil d'un centre documentaire multimedia

22 (1976), no 2, p. 95-97

(Larivière, Jules). Hébert, Françoise. La photocopie dans les bibliothèques canadiennes

34 (1988), no 3, p. 111-112

(Larose, Hélène). Houle, Ghislaine. La femme et la société québécoise

21 (1975), no 3, p. 169-171

Laserre, Béatrice. La lecture publique en Suisse romande : enquête du groupe de travail des bibliothèques de lecture publique, par Béatrice Laserre et Catherine Tacchini. (Réal Messier)

25 (1979), no 4, p. 225-227

(Lassonde, Jean-René). Les livres disponibles $1978=$ French Books in Print

25 (1979), no 4, p. 219-221

Lassonde, Jean-René. La bibliothèque Saint-Sulpice, 1910-1931. (Ginette Vadnais)

33 (1987), no 2, p. $72-73$

(Latreille-Huvelin, France). Tétreault, Raymond. Le livre dans la vie de l'enfant. Actes du Colloque 1977. Textes colligés et présentés par Raymond Tétreault

27 (1981), no 1, p. 36-37

(Latreille-Huvelin. France). Potvin, Claude. Le Canada français et sa littérature de jeunesse

29 (1983), no 2, p. 79

Laurentiana parus avant 1821

voir Québec. Bibliothèque nationale du Québec

Lauzier, Suzanne. Les ouvrages de référence du Québec. Supplément 19671974, par Suzanne Lauzier et Normand Cormier. (V. Alexandre Jonynas)

22 (1976), no 2, p. 91-93

(Lavergne, Rodolphe). Scientific and Technical Societies of Canada / Sociétés scientifiques et techniques du Canada ( $2 e$ éd.)

19 (1973), no 1, p. 42

Laverne Carroll, Frances

voir Carroll, Frances Laverne 
(Lavigne, Nicole). DSI/CB, Service de diffusion sélective de l'information de la Centrale des bibliothèques

31 (1985), no 1, p. $39-40$

(Lavigne, Nicole). Franklin, Karen. Un titre s'il vous plait : conseils à l'intention des éditeurs de revues spécialisées, rapports, journaux et autres publications en série canadiennes

31 (1985), no 2, p. 73-74

(Lavigne, Nicole). Union List of Serials in the Montreal Area = Catalogue collectif des périodiques de la région de Montréal. 1986

32 (1986), no 1-2, p. 57-58

(Le Deuff, Marylène). Chaumier, Jacques. Travail et méthodes du / de la documentaliste

28 (1982), no 1, p. $33-35$

(LeBel, Clément). Règles de catalogage anglo-américaines. (Deuxième édition, révision de 1988)

37 (1991), no 2, p. 81-82

(Lebel-Paquin, Denise). Classe PS 8000. Cadre de classification de la littérature canadienne ( 2 e éd.)

25 (1979), no 1, p. 50-51

Lecompte, Luc-Louis. Thésaurus : Enfance inadaptée, par Pierrette Dubuc, Lise Thibodeau-Brunet et Luc-Louis Lecompte. (Jean Bouthillette et Raymond Caron)

27 (1981), no 2, p. 82-83

La lecture publique en Suisse romande : enquête du groupe de travail des bibliothèques de lecture publique

voir Laserre, Béatrice

(Lefebvre, Gilbert). Day, Mario. Accès aux publications gouvernementales québécoises et canadiennes : index permuté 25 (1979), no 3, p. 161-162

(Lefebvre, Gilbert). Thériault, Yvon. Les publications parlementaires d'hier et d'aujourd'hui. 2e éd.

30 (1984), no 2, p. 61

Legris, Renée. Répertoire des dramatiques québécoises à la télévision, 1952-1977, par Pierre Pagé et Renée Legris. (Michel Boisvert)

$$
24 \text { (1978), no 2, p. } 91
$$

Legris, Renée. Répertoire des oeuvres de la littérature radiophonique québécoise, 1930-1970, par Pierre Pagé, avec la collaboration de Renée Legris et Louise Blouin. (Jean-Pierre Chalifoux)

$$
21 \text { (1975), no 3, p. 171-172 }
$$

Lemaître, Renée. Drôles de bibliothèques... : le thème de la bibliothèque dans la littérature et le cinéma, par Anne-Marie Chaintreau et Renée Lemaître. (Jacques Paradis)

$$
37 \text { (1991), no 1, p. 44-46 }
$$

Lemire, Maurice et al. La vie littéraire au Québec. (Claude Fournier)

37 (1991), no 4, p. 163-164

Lemire, Maurice, éd. Dictionnaire des oeuvres littéraires du Québec. Tome 1 : des origines à 1900. (Jean-Pierre Chabot)

25 (1979), no 1, p. 53-56

Lemoine, Roger. Catalogue de la bibliothèque de Louis-Joseph Papineau. (Gilles Gallichan)

$$
29 \text { (1983), no 4, p. } 167
$$

(Lépine, Pierre). Centre d'études en enseignement du Canada. L'inter Atlas : les ressources du Québec et du Canada

$$
34 \text { (1988), no 3, p. 110-111 }
$$

(Lépine, Pierre). Tessier, Yves, comp. Répertoire des atlas de la cartothèque, compilé par Yves Tessier

$$
20 \text { (1974), no 2, p. 106-107 }
$$

(Létourneau, Hugues). Tamaro, Normand. La loi sur le droit d'auteur commentée et annotée

$$
38 \text { (1992), no 3, p. } 171
$$

(Léveillé, Yves). Services documentaires multimedia inc. Listes bibliographiques spécialisées correspondantes aux programmes d'études de l'enseignement primaire et secondaire

$$
36 \text { (1990), no 2, p. } 78-80
$$

Lévesque, Albert. Contribution to the National Bibliography of Rwanda : 19651970. Contribution à la bibliographie nationale du Rwanda. (Victor Coulombe)

26 (1980), no 3, p. 173-177
Lévesque, Raymond. La bibliographie d'un plan de cours de niveau collégial et les paradigmes éducationnels pour une nouvelle définition et un nouveau cadre théorique de la bibliographie. (Bernhard, Paulette)

34 (1988), no 3, p. 113-115

Lexicon of Information and Documentation voir Bonin, Claude-André

Lexique d'information documentaire = Lexicon of Information and Documentation voir Bonin, Claude-André

Libère, François. Le livre, mutations actuelles. (Jean-Rémi Brault) 36 (1990), no 4, p. 141

La librairie et l'édition à Montréal, 17761920

voir Lamonde, Yvan

Library of Congress

voir Etats-Unis. Library of Congress

Lire à "Bordeaux"

voir Castonguay, André

Lise et Bruno dans l'univers des livres voir Mignault, Marcel

Liste mensuelle des publications du gouvernement du Québec. (Gaston Bernier)

30 (1984), no 1, p. $28-29$

Liste rotative de descripteurs sur l'éducation

voir Service coopératif de transmission de l'information sur l'éducation

Listes bibliographiques spécialisées correspondantes aux programmes d'études de l'enseignement primaire et secondaire voir Services documentaires multimedia inc.

Literary and Journalistic Awards in Canada I Les prix de littérature et de journalisme au Canada, 1923-1973

voir Canada. Statistique Canada. Division de l'éducation, des sciences et de la culture. Section des beaux-arts et des médias

Littérature québécoise et cinéma voir Revue d'histoire littéraire du Québec et du Canada

Le livre dans la vie de l'enfant. Actes du Colloque 1977

voir Tétreault, Raymond 
Le livre et la construction de la personnalité de l'enfant

voir Mareuil, André

Livre et lecture au Québec (1800-1850), sous la direction de Claude Galarneau et Maurice Lemire. (Jean-Rémi Brault)

$$
34 \text { (1988), no 4, p. } 159-160
$$

Livre et politique au Bas-Canada, 17911849

voir Gallichan. Gilles

Le livre français : hier, aujourd'hui, demain. voir Cain, Julien

Le livre français a-t-il un avenir ?

voir Cahart, Pierre

Le livre religieux au Québec depuis les débuts de l'imprimerie jusqu'à la Confédération (1764-1867)

voir Thériault, Michel

Livre, bibliothèque et culture québécoise. Mélanges offerts à Edmond Desrochers voir Chartrand, Georges A.

Le livre, mutations actuelles

voir Libère, François

Livres africains disponibles

voir Zell, Hans M.

Livres blancs et livres verts au Québec, 1964-1981

voir Deschênes, Gaston

Les livres disponibles $1978=$ French Books in Print. (Jean-René Lassonde)

25 (1979), no 4, p. 219-221

Livres en langue française pour les jeunes voir Charbonneau, Hélène

La loi sur le droit d'auteur commentée et annotée

voir Tamaro, Normand

(Lord, Jules). Infodex : index de la Presse

33 (1987), no 1, p. 32-34

Lorsque notre littérature était jeune. Entretiens avec Pierre Tisseyre

voir Guay, Jean-Pierre

Lurelu : bulletin d'information sur la littérature de jeunesse (vol.1, no 1-). (JeanPierre Chabot)

26 (1980), no 2, p. 119-122
Luthi, J.J. Dictionnaire général de la francophonie, par J.J. Luthi, A. Viatte, G. Zanarini. (Gilles Gallichan)

34 (1988), no 2, p. $79-80$

M

(MacKay, Louise). Lamy-Rousseau, Françoise. Traitement automatisé des documents multimedia avec les systèmes ISBD unifié, Lamy-Rousseau et PRECIS. Propositions SILP

21 (1975), no 4, p. 229-231

Macrothésaurus pour le traitement de l'information relative au développement économique et social ( $4 \mathrm{e}$ éd.). (Rénald Buteau)

37 (1991), no 4, p. 167

(Magnan, Fernand C.). L'architecture française : recherche - formes intérieures arts - urbanisme (...) nos 369-370

20 (1974), no 1, p. $48-50$

Maillet, Lise. Provincial Royal Commissions and Commissions of Inquiry, 18671982 : A Selective Bibliography = Commissions royales provinciales et commissions d'enquête, 1867-1982 : bibliographie sélective. (Gaston Bernier) 32 (1986), no 3, p. 102-103

(Mailloux, Monique). Bernier, Gérald. Le Québec en transition : 1760-1867 : bibliographie thématique, par Gérald Bernier et Robert Boily

34 (1988), no 4 , p. $160-162$

Mailloux, Pierre, comp. Bibliographie annotée d'ouvrages de référence en usage au bureau de la bibliographie rétrospective. (Jacques Sansfaçon)

20 (1974), no 4, p. 206-207

(Malette, Danielle). Breton, Jean et al. Guide pratique d'indexation en vocabulaire libre

30 (1984), no 2, p. $63-64$

(Malette, Danielle). Le monde de la documentation et les pouvoirs publics

27 (1981), no 3, p. 123-126

(Manseau, Pierre). Lamy-Rousseau, Françoise. Uniformisation des règles de catalogage des documents visuels et sonores: description d'une expérience, par Françoise Lamy-Rousseau. En collab. avec Martine Paquet.

20 (1974), no 1 , p. $50-51$
(Manseau,Pierre). American Library Association. Règles de catalogage angloaméricaines (2e éd.)

26 (1980), no 4, p. $218-220$

Manuel des procédures du prêt entre bibliothèques

voir Services de prêt entre bibliothèques : manuel d'information

Manuel du bibliothécaire documentaliste travaillant dans les pays en développement voir Association française des documentalistes et bibliothécaires spécialisés

Mareuil, André. Le livre et la construction de la personnalité de l'enfant. (Marielle Durand)

24 (1978), no 3, p. 152-154

Maria Chapdelaine : évolution de l'édition 1913-1980

voir Cormier, Normand

(Marquis, Julien). Répertoire de vedettesmatière. 9e éd.

30 (1984), no 3, p. 105-106

(Martin, Andrée). Houle, Ghislaine. La femme et la société québécoise

21 (1975), no 3, p. 169-171

(Martin, Andrée). Québec (Province) Conseil du statut de la femme. Les Québécoises : guide bibliographique suivi d'une filmographie

23 (1977), no 1, p. 45-47

Martin, Henri-Jean. Le livre français : hier, aujourd'hui, demain. Ouvrage collectif sous la direction de Julien Cain, Robert Escarpit, Henri-Jean Martin. (Jean-Louis Roy)

20 (1974), no 1, p. 44-45

Masson, Annie Decroux voir Decroux-Masson, Annie

(Mathieu, Régis). Fournier-Renaud, Madeleine. Ecrits sur le cinéma. bibliographie québécoise 1911-1981, par Madeleine Fournier-Renaud et Pierre Véronneau

30 (1984), no 3, p. 103-104

Melet-Sanson, Jacqueline. La Bibliothèque nationale, mémoire de l'avenir, par Bruno Blasselle et Jacqueline Melet-Sanson. (Jean-Rémi Brault)

37 (1991), no 2, p. $84-85$

Melot, Michel. Propositions pour une grande bibliothèque, par Patrice Cahart et Michel Melot. (Jean-Rémi Brault)

36 (1990), no 3, p. 111-112 
Mémoire sur une politique du livre et de la lecture au Québec présenté à Monsieur Louis O'Neill, ministre des Affaires culturelles

voir Québec. Comité consultatif du livre

Mennie-de Varennes, Kathleen. Bibliographie annotée d'ouvrages généalogiques au Canada = Annotated Bibliography of Genealogical Works in Canada. (JeanRémi Brault)

33 (1987), no 4, p. 138

Menou, Michel. Introduction générale aux sciences et techniques de l'information et de la documentation. par Claire Guinchat et Michel Menou. (Suzanne BertrandGastaldy)

29 (1983), no 1, p. $41-43$

(Messier, Réal). Bergeron, Alain. Les habitudes de lecture des québécois 20 (1974), no 1, p. $47-48$

(Messier, Réal). Germanaud, Marie-Claire. La bibliothèque publique en milieu rural et dans les petites agglomérations

29 (1983), no 4, p. 169

(Messier, Réal). Héon, Gérard. Les habitudes de lecture des québécois de 10-12 ans

29 (1983), no 2 , p. $79-80$

(Messier, Réal). Laserre, Béatrice. La lecture publique en Suisse romande : enquête du groupe de travail des bibliothèques de lecture publique, par Béatrice Laserre et Catherine Tacchini

25 (1979), no 4, p. 225-227

(Messier, Réal). Ouellet, Jacques. La situation des bibliothèques publiques au Saguenay/Lac Saint-Jean

33 (1987), no 3, p. 103-104

Mesure de la performance dans les bibliothèques fédérales : un guide. Performance Measurement in Federal Libraries: A Handbook

voir Canada. Comité des systèmes unifiés de bibliothèque. Groupe de travail sur la mesure de la performance

Méthodologie d'utilisation d'un centre de documentation

voir Chevalier, Brigitte

(Meunier, Claire). Bourron, Yves. Audiovisuel, pédagogie et communication

28 (1982), no 4 , p. $169-170$
Mignault, Marcel. Lise et Bruno dans l'univers des livres. (Denyse Bourneuf)

23 (1977), no 3, p. 173-174

Ministère de l'Education. Direction générale de l'administration

voir Québec. Ministère de l'Education. Direction générale de l'administration

Miquel, André. Les bibliothèques universitaires. Rapport au ministre d'État, ministre de l'Éducation nationale, de la Jeunesse et des Sports. (Céline Cartier)

36 (1990), no 2, p. 75-77

Mittermeyer, Diane. Les bibliothèques publiques canadiennes de 1979 à 1983 : analyse comparative de certaines données statistiques. (Jean-Rémi Brault)

33 (1987), no 4, p. $139-140$

Le monde de la documentation et les pouvoirs publics. (Danielle Malette)

27 (1981), no 3, p. 123-126

(Mongeau, Lise). Nobréga, Gérard. Thésaurus Envirodoq, en collaboration avec Informatech France-Québec.

27 (1981), no 3, p. 130-132

Monière, Denis. Les idéologies au Québec, par Denis Monière et André Vachet. (Daniel Rémi)

23 (1977), no 1 , p. 51-52

(Morin, Marc). Bélanger, Nycole. "La documentation", Méta (numéro spécial), v.25, no 1, (mars 1980), sous la direction de Nycole Bélanger

27 (1981), no 1, p. $37-39$

Morin, Marie-Josée. L'indispensable en documentation; les outils de travail, par Manon Bourget, Robert Chiasson et MarieJosée Morin. (Leonard Nadeau)

37 (1991), no 1, p. 43-44

Multi-Réso Inc. La presse écrite au Québec. Bilan et prospective. (Jean de Bonville)

25 (1979), no 3, p. 157-161

Multi-Réso Inc. Une agence de presse au Québec? Nécessité, utilité et viabilité économique (Rapport). (Jean de Bonville)

25 (1979), no 3, p. 157-161
N

Nabhan, Victor. Problèmes de droit d'auteur en éducation, par Christian Vincke. Avec la collaboration de Pierre A. Côté et Victor Nabhan. (Claudio Antonelli) 25 (1979), no 1, p. $49-50$

(Nadeau, Léonard). Bourget, Manon. L'indispensable en documentation; les outils de travail, par Manon Bourget, Robert Chiasson et Marie-Josée Morin

37 (1991), no 1, p. 43-44

(Nadeau, Léonard). Calvé-Woodrough, Lise. Recueil d'exemples illustrant les Règles de catalogage anglo-américaines. Edition provisoire sous la direction de Lise Calvé-Woodrough et Michel Faubert. Avec la collaboration de Louise Gagnon (et autres)

21 (1975), no 4, p. 231-232

Neale, Colin. Projet d'établissement d'un système d'information et de distribution pour les produits audiovisuels canadiens. Proposed Course of Action to Implement an Information Distribution System for Canadian Audio-Visual Products. (Jacques Demers)

24 (1978), no 4 , p. $205-208$

(Nkembé, Théo). Zell, Hans M. African Books in Print. Livres africains disponibles.

26 (1980), no 4, p. 224-225

Nobréga, Gérard. Thésaurus Envirodoq, en collaboration avec Informatech FranceQuébec. (Lise Mongeau et Diane Sauvé)

27 (1981), no 3, p. 130-132

Normalisation du catalogage

voir Canada. Groupe de travail sur la normalisation du catalogage

Normes pour les bibliothèques municipales voir Québec. Ministère des Affaires culturelles. Senvice des bibliothèques publiques

Nouveau dictionnaire des difficultés du français moderne

voir Hanse, Joseph

O

O.I.T.

voir Bureau international du travail 
Office des personnes handicapées du Québec. 300 documents audiovisuels sur les personnes handicapées. (Jean-Rémi Brault)

35 (1989), no 1, p. 29-30

Olier, J. H. d'. La planification des infrastructures nationales de documentation, de bibliothèques et d'archives, par J. $\mathrm{H}$. d'Olier et B. Delmas. (Réal Bosa)

22 (1976), no 1, p. 50-51

Olivier, Daniel. Les bibliothèques personnelles au Québec : inventaire analytique et préliminaire des sources par Yvan Lamonde et Daniel Olivier. (Gilles Gallichan)

29 (1983), no 4, p. 167

Oster, Daniel. Dictionnaire de littérature française contemporaine, par Claude Bonnefoy, Tony Cartano et Daniel Oster. (Patrick Coppens)

24 (1978), no 3, p. 146-148

Ouellet, Jacques. La situation des bibliothèques publiques au Saguenay/Lac SaintJean. (Réal Messier)

33 (1987), no 3, p. 103-104

(Ouimet, Laurent). Québec. Bibliothèque de la Législature. Index des lois à caractère privé du Québec, 1867-1975

24 (1978), no 2, p. 95

L'Outaouais québécois. Guide de recherche et bibliographie sélective

voir St-Amour, Jean-Pierre F.

Les ouvrages de référence du Québec : supplément analytique

voir Boivin, Henri-Bernard

Les ouvrages de référence du Québec. Supplément 1967-1974

voir Québec. Bibliothèque nationale

Ouvrages de référence pour les bibliothèques publiques : répertoire bibliographique

voir Beaudiquez, Marcelle

Ouvrages de référence sur la musique et les phonogrammes. Guide à l'usage des bibliothécaires et discothécaires

voir Alix, Yves

\section{$\mathbf{P}$}

Pagé, Pierre. Répertoire des dramatiques québécoises à la télévision, 1952-1977, par Pierre Pagé et Renée Legris. (Michel Boisvert)

$$
24 \text { (1978), no 2, p. } 91
$$

Pagé, Pierre. Répertoire des oeuvres de la littérature radiophonique québécoise, 1930-1970, par Pierre Pagé, avec la collaboration de Renée Legris et Louise Blouin. (Jean-Pierre Chalifoux)

$$
21 \text { (1975), no 3, p. 171-172 }
$$

Panaccio, Colette

voir Rivet-Panaccio, Colette

Panaccio, Colette Rivet

voir Rivet-Panaccio, Colette

(Panneton, Jacques). Québec. Ministère des Affaires culturelles. Service des bibliothèques publiques. Normes pour les bibliothèques municipales

$$
21 \text { (1975), no 3, p. 167-169 }
$$

(Panneton, Jacques). Une bibliothèque pour Montréal; rapport préléminaire sur la possibilité d'établir une bibliothèque publique à la Place Desjardins, sous la direction de André Castonguay, par JeanMarc Alain. (et autres)

$$
20 \text { (1974), no 1, p. } 42-43
$$

Papa lit, maman coud; les manuels scolaires en bleu et rose

voir Decroux-Masson, Annie

(Papillon, Yvon). Canada. Archives publiques. Plan de conservation et d'élimimation des documents

28 (1982), no 3, p. 132-133

Paquet, Martine. Uniformisation des règles de catalogage des documents visuels et sonores : description d'une expérience, par Françoise Lamy-Rousseau. En collab. avec Martine Paquet. (Pierre Manseau)

$$
20 \text { (1974), no 1, p. 50-51 }
$$

Paquin, Denise Lebel

voir Lebel-Paquin, Denise

(Paradis, Jacques). Bulletin du livre. no 11958-

19 (1973), no 3, p. 134-136
(Paradis, Jacques). Chaintreau, AnneMarie. Drôles de bibliothèques... : le thème de la bibliothèque dans la littérature et le cinéma, par Anne-Marie Chaintreau et Renée Lemaître

37 (1991), no 1, p. $41-46$

(Paradis, Jacques). Connaissance et formation par le livre et l'audiovisuel. no.11973-

$$
20 \text { (1974), no 1, p. } 45-47
$$

(Paradis, Jacques). Francophonie édition. no 1- nov. 1972-

19 (1973), no 3, p. 134-136

(Paradis, Jacques). Le Tessier; répertoire des documents audiovisuels canadiens de langue française

30 (1984), no 1, p. $30-31$

(Paradis, Jacques). Répertoire de l'édition au Québec. (1re éd.) 1972-

19 (1973), no 3, p. 134-136

(Paradis, Jacques). Répertoire des livres de langue française disponibles (1re éd.) 1972-

19 (1973), no 3, p. 134-136

(Paradis, Jacques). Viens de paraître. v. 11965-

19 (1973), no 3, p. 134-136

Paralittérature 1

voir Allard, Yvon

Parchemin s'explique...; guide de dépouillement des actes notariés du Québec ancien

voir Lafortune, Hélène et al.

(Paré, Gilles). Sources d'information sur la consommation

23 (1977), no 1, p. 52-54

(Paré, Richard). Bibliothèque nationale du Canada. Répertoire des bibliothèques canadiennes. 1- Bibliothèques du gouvernement fédéral / Canadian Library Directory. 1- Federal Government Libraries

20 (1974), no 4, p. 208-209

(Paré, Richard). Guinchat, Claire. Guide pratique des techniques documentaires par Claire Guinchat et Yolande Skouri avec la collaboration de Marie-Pierre Alix 37 (1991), no 2, p. 83

(Paul, André). Dewey, Melvil. Classification décimale de Dewey et index, établis par Melvil Dewey

21 (1975), no 1, p. 60-62 
La pédagogie de la documentation dans le contexte de l'audiovisuel et des massmédia

voir Faurie, Albertine

(Pellerin, Maurice). Fortier, Marie-France. Index du bulletin des recherches historiques

35 (1989), no 1, p. 28

(Pelletier, Rosaire). Bélanger, Marcel. L'utilisation des moyens didactiques imprimés et audiovisuels par les professeurs au secondaire : une étude préliminaire, par Marcel Bélanger et Hoa-Thuy Pham-Dang

23 (1977), no 3, p. 171-173

(Pelletier, Rosaire). Bergeron, Christian. Les centres de ressources éducatives dans l'enseignement secondaire : exploration de la diversité des modèles, des situations et des problèmes locaux, par Christian Bergeron et Hoa-Thuy Pham-Dang

23 (1977), no 3, p. 171-173

(Pelletier, Rosaire). Béthery, Annie. Abrégé de la classification décimale de Dewey. Nouvelle édition entièrement refondue et complétée à partir de la première version intégrale française et de la XIXe édition intégrale en langue anglaise

29 (1983), no 4, p. 165

(Pelletier, Rosaire). Reboul, Elie. Information et pédagogie

24 (1978), no 4, p. 211-212

Pelou, Pierre. L'Europe de l'information. (Suzanne Richer)

36 (1990), no 3, p. 110-111

Performance Measurement in Federal Libraries: A Handbook

voir Canada. Comité des systèmes unifiés de bibliothèque. Groupe de travail sur la mesure de la performance

Periodex, index analytique de périodiques de langue française

voir Centrale des bibliothèques

Les périodiques

voir Bethery, Annie

Périodiques pour les collèges

voir Centrale des bibliothèques. Centre de bibliographie

Perol, Dominique. Initiation à la bibliographie scientifique, par Marie-France Such et Dominique Perol. (Claude Busque) 35 (1989), no 1, p. 28-29
(Perreault, Micheline). Alix, Yves. Ouvrages de référence sur la musique et les phonogrammes. Guide à l'usage des bibliothécaires et discothécaires.

29 (1983), no 4, p. 169

(Perrier, Alain). Les années '80. au service des clientèles

28 (1982), no 3, p. 133-135

(Perrier, Alain). Québec. Comité consultatif du livre. Mémoire sur une politique du livre et de la lecture au Québec présenté à Monsieur Louis O'Neill, Ministre des Affaires culturelles

24 (1978), no 1, p. $37-40$

(Perron, Hubert). Chartrand, Georges A. Livre, bibliothèque et culture québécoise. Mélanges offerts à Edmond Desrochers, sous la direction de Georges A. Chartrand

25 (1979), no 2, p. 101-103

Petit dictionnaire des écrivains

voir Union des écrivains québécois

La photocopie dans les bibliothèques canadiennes

voir Hébert, Françoise

Plan de conservation et d'élimimation des documents

voir Canada. Archives publiques

La planification des infrastructures nationales de documentation, de bibliothèques et d'archives

voir Olier, J. H. d'

(Plante, Jean-Paul). Information et documentation : attente des pays en voie de développement. Information and Documentation. The Expectations of Developing Countries. Actes du Colloque organisé par le comité des relations internationales de l'ASTED

$$
29 \text { (1983), no 3, p. 121-122 }
$$

Point de repère : index analytique d'articles de périodiques québécois et étrangers. (Jean-Luc Fortin)

31 (1985), no 1, p. 41-43

Poole, Erik. Guerre, paix et désarmement. Bibliographie thématique en langue française, par Érik Poole et Annie Bourret. (Rénald Buteau)

36 (1990), no 3, p. 108-109
Poole, Érik. Index sur la guerre et la paix : index relationnel de mots-clés pour SONJA, banque de données informatisées sur la guerre, la paix et le désarmement en langue française, par Annie Bourret et Érik Poole. (Rénald Buteau)

36 (1990), no 3, p. 108-109

(Potin, Monique). Groupe de travail sur l'évaluation des bibliothèques de collèges. L'évaluation de l'efficacité de la bibliothèque de collège : une approche systémique

26 (1980), no 1, p. $49-51$

Potvin, Claude. Le Canada français et sa littérature de jeunesse. (France LatreilleHuvelin)

29 (1983), no 2, p. 79

Pour une gestion adaptée des services documentaires

voir Alain, Jean-Marc

Pour une médiathèque : guide de l'audiovisuel à l'usage des candidats aux concours et examens des bibliothèques voir Herzhaft, Gérard

Pour une politique québécoise de la recherche scientifique

voir Québec. Ministère d'Etat au Développement culturel

(Powell, Wyley L.) Prospectives en bibliothéconomie et en sciences de l'information: mélanges dédiés à la mémoire de Laurent-G. Denis. Réunis sous la direction de Paule Rolland-Thomas

37 (1991), no 1, p. 41-42

La presse écrite au Québec voir Multi-Réso Inc.

La presse québécoise des origines à nos jours, 1920-1934, 1935- 1944

voir Beaulieu, André

La presse québécoise des origines à nos jours. Tome premier : 1764-1850 voir Beaulieu, André

Prévenir ou guérir?

voir Colloque sur la conservation des collections Montréal, 16-17 novembre 1989

Principes directeurs pour l'enseignement du marketing dans la formation des bibliothécaires, documentalistes et archivistes voir Savard, Réjean 
Principes directeurs pour la rédaction d'objectifs d'apprentissage en bibliothéconomie, en sciences de l'information et en archivistique

voir Fontaine, France

Les prix de littérature et de journalisme au Canada, 1923-1973

voir Canada. Statistique Canada. Division de l'éducation, des sciences et de la culture. Section des beaux-arts et des médias

Problèmes de droit d'auteur en éducation voir Vincke, Christian

Proceedings of the National Bibliographies Seminar, hold under the auspices of the IFLA Division of Bibliographic Control. (Louise Filion)

36 (1990), no 4, p. 142-143

Profil d'un centre documentaire multimedia voir Szpakowska, Janina-Klara

Profils géo-documentaires des États francophones du Sud. (Troisième édition rev. et augm.) (Bernier, Gaston)

38 (1992), no 3, p. 173-174

Projet d'établissement d'un système d'information et de distribution pour les produits audiovisuels canadiens

voir Neale, Colin

Projet progrès : étude sur les bibliothèques publiques canadiennes. (Jean-Guy Desroches)

28 (1982), no 1 , p. $36-38$

A Proposal for a Selective, Annotated Catalogue of Canadian Produced NonPrint Materials

voir Rothwell, Hélène de F.

Propositions pour une grande bibliothèque voir Cahart Patrice

Prospectives en bibliothéconomie et en sciences de l'information : mélanges dédiés à la mémoire de Laurent-G. Denis. Réunis sous la direction de Paule RollandThomas. (Wyley L. Powell)

37 (1991), no 1, p. $41-42$

Provincial Royal Commissions of inquiry, 1867-1982 : a selective bibliography = Commissions royales provinciales et commissions d'enquête, 1867-1982 bibliographie sélective

voir Maillet, Lise
Les publications parlementaires d'hier et d'aujourd'hui. 2e éd.

voir Thériault, Yvon

Publications parlementaires québécoises: catalogue collectif

voir Gagné, Raymond

La publicité en action : comment élaborer une campagne de publicité ou ce qui se passe derrière les murs d'une agence voir Cossette, Claude

\section{Q}

Québec (Province). Conseil du statut de la femme. Les Québécoises : guide bibliographique suivi d'une filmographie. (Andrée Martin)

23 (1977), no 1 , p. $45-47$

Québec (Province). Ministère des Communications. Direction générale de l'édition. Centre de documentation. Répertoire analytique des publications gouvernementales

23 (1977), no 3, p. $170-171$

Le Québec en chiffres de 1850 à nos jours voir Bernier, Gérald

Le Québec en transition : 1760-1867: bibliographie thématique

voir Bernier, Gérald

Le Québec mis en chiffres : statistiques sociales et économiques sur les régions et les villes du Québec

voir Fabre, Jean-Bernard

Le Québec statistique, 1985-1986. 58e éd. (Roger de la Sablonnière)

$$
32 \text { (1986), no 3, p. 98-99 }
$$

Québec. Archives nationales. Guide. Archives écrites d'origine privée conservées au Centre d'archives de Québec et de Chaudière-Appalaches. (Gilles Gallichan)

38 (1992), no 3, p. 174-175

Québec. Assemblée nationale. Débats de l'Assemblée législative, 1867-1878, 18931901. (Marcel Caya)

30 (1984), no 2, p. $67-68$

Québec. Assemblée nationale. Journal des débats. Index 1988/1989. (Suzanne Bertrand-Gastaldy)

$$
37 \text { (1991), no } 4 \text {, p. } 167-168
$$

Québec. Bibliothèque de la législature. Index des lois à caractère privé du Québec 1867-1975. (Laurent Ouimet)

24 (1978), no 2, p. 95

Québec. Bibliothèque de la législature. Le référendum : bibliographie sélective et annotée. (Lise Wilson)

24 (1978), no 1, p. $43-44$

Québec. Bibliothèque nationale

voir aussi Bibliothèque nationale $\mathrm{du}$ Québec

Québec. Bibliothèque nationale du Québec. Bibliographie de bibliographies québécoises. (Jean-Pierre Chalifoux)

26 (1980), no 1, p. 51-53

Québec. Bibliothèque nationale du Québec. Laurentiana parus avant 1821, par Milada Vlack, avec la collaboration de Yolande Buono

23 (1977), no 4, p. 215-216

Québec. Bibliothèque nationale du Québec. Statistiques de l'édition au Québec en 1990. (Sylvie Faure)

38 (1992), no 4, p. 214-215

Québec. Bibliothèque nationale. Les ouvrages de référence du Québec. Supplément 1967-1974. (V. Alexandre Jonynas)

22 (1976), no 2, p. 91-93

Québec. Bureau de la statistique du Québec. Répertoire de données et de publications statistiques québécoises. (Roger de la Sablonnière)

30 (1984), no 2, p. 62-63

Québec. Comité consultatif du livre. Mémoire sur une politique du livre et de la lecture au Québec présenté à Monsieur Louis O'Neill, Ministre des Affaires culturelles. (Alain Perrier)

24 (1978), no 1 , p. $37-40$

Québec. Comité d'étude d'un réseau des bibliothèques québécoises pour le traitement en coopération de leur documentation. Vers un réseau québécois des bibliothèques. Rapport du CERBIQ. (Eric de Grolier)

25 (1979), no 4, p. 217-219

Québec. Ministère d'État au Développement culturel. Pour une politique québécoise de la recherche scientifique. (JeanMarc Alain)

26 (1980), no 2 , p. 113-116 
Québec. Ministère de l'Éducation. Direction générale de l'administration. Guide d'aménagement des bibliothèques-centres documentaires des écoles élémentaires. (Daniel Reicher)

$$
24 \text { (1978), no 1, p. 47-48 }
$$

Québec. Ministère de l'Éducation. Direction générale de l'évaluation et des ressources didactiques. Direction des ressources didactiques. Les bibliothèques scolaires québécoises. Plus que jamais... (Jean-Paul Roy)

$$
35 \text { (1989), no 4, p. 189-190 }
$$

Québec. Ministère des Affaires culturelles. Service des bibliothèques publiques. Normes pour les bibliothèques municipales. (Jacques Panneton)

$$
21 \text { (1975), no 3, p. 167-169 }
$$

Québec. Ministère des Communications. Direction générale du Développement et des Politiques. De la précarité de la presse ou le citoyen menacé. Rapport du comité de travail sur la concentration de la presse écrite. (Jean de Bonville)

$$
25 \text { (1979), no 3, p. 157-161 }
$$

Les Québécoises : guide bibliographique suivi d'une filmographie

voir Québec (Province). Conseil du statut de la femme

\section{$\mathbf{R}$}

Radar, répertoire analytique d'articles de revues du Québec

voir Bibliothèque nationale du Québec

Raiffaud, Joël. La gestion documentaire, par Joël Raiffaud et associés. (Danièle Daoust)

30 (1984), no 4, p. 144-145

Rapport

voir Bibliothèque nationale du Canada. Groupe de travail sur le service de bibliothèque aux handicapés

voir Bibliothèque nationale du Canada. Groupe de travail sur le catalogue collectif canadien. Sous groupe de travail sur les catalogues collectifs de publications en série

Rapport de la Commission d'étude sur l'accès du citoyen à l'information gouvernementale et sur la protection des renseignements personnels

voir Information et liberté. Rapport de la...
Rapport de la consultation du ministre des Affaires culturelles du Québec. (Gabriel Dussault)

$$
30 \text { (1984), no 2, p. } 64-67
$$

Rapport de recherche sur les bibliothèques scolaires et l'enseignement élémentaire dans la région de l'Estrie voir Gouvernement du Québec

Rapport du Comité d'étude de la politique culturelle fédérale. (Gabriel Dussault) 30 (1984), no 2, p. 64-67

Rapport du comité de travail sur la concentration de la presse écrite

voir Québec. Ministère des Communications. Direction générale du Développement et des Politiques

Rapport final soumis au directeur général de la Bibliothèque nationale

voir Bibliothèque nationale du Canada. Groupe de travail sur le catalogue collectif canadien

\section{A Reader's Guide to Québec Studies voir Sénécal, André}

Reboul, Elie. Information et pédagogie. (Georgette Lapointe et Rosaire Pelletier) 24 (1978), no 4, p. 211-212

Reboul, Jacquette. Du bon usage des bibliographies. (Louise Beauregard)

$$
20 \text { (1974), no 4, p. 207-208 }
$$

Recueil d'exemples illustrant les Règles de catalogage anglo-américaines voir Calvé-Woodrough, Lise

Le référendum : bibliographie sélective et annotée voir Québec. Bibliothèque de la législature

Règles de catalogage anglo-américaines voir Rolland-Thomas, Paule

Règles de catalogage anglo-américaines. (2e éd.)

voir American Library Association

Règles de catalogage anglo-américaines. (Deuxième édition, révision de 1988). (Clément LeBel)

37 (1991), no 2, p. 81-82

(Reicher, Daniel). Québec. Ministère de l'Education. Direction générale de l'administration. Guide d'aménagement des bibliothèques-centres documentaires des écoles élémentaires

24 (1978), no 1, p. 47-48
(Rémi, Daniel). Monière, Denis. Les idéologies au Québec, par Denis Monière et André Vachet

23 (1977), no 1, p. 51-52

Renaud, Madeleine Fournier

voir Fournier-Renaud, Madeleine

(Renaud-Frigon, Claire). Service coopératif de transmission de l'information sur l'éducation. Liste rotative de descripteurs sur l'éducation

19 (1973), no 1, p. $45-46$

Renyi, Pierre. Dictionnaire anglais-français de l'électronique et de l'électrotechnique, par Pierre Renyi et Dominique Amrouni. (Claude Fournier)

33 (1987), no 1, p. $29-30$

La répartition du budget des collections au Service des bibliothèques de l'Université de Sherbrooke

voir Gaudette, Pierre

Répertoire analytique des publications gouvernementales

voir Québec (Province). Ministère des Communications. Direction générale de l'édition. Centre de documentation

Répertoire cartobibliographique de Charlevoix, [compilé par] Daniel Dufour. (Yves Tessier)

33 (1987), no 2, p. 74-76

Répertoire de données et de publications statistiques québécoises

voir Québec. Bureau de la statistique du Québec

Répertoire de l'édition au Québec. (1re éd.) 1972- . (Jacques Paradis)

19 (1973), no 3, p. 134-136

Répertoire de vedettes-matière voir Université Laval. Bibliothèque. Service de catalogage

Répertoire de vedettes-matière. $9 e$ éd. (Julien Marquis)

30 (1984), no 3, p. 105-106

Répertoire des atlas de la cartothèque voir Tessier, Yves

Répertoire des bibliothèques canadiennes. 1- Bibliothèques du gouvernement fédéral / Canadian Library Directory. 1- Federal Government Libraries voir Bibliothèque nationale du Canada 
Répertoire des centres de documentation et des bibliothèques spécialisées et de recherche, 1992. (Jean-Rémi Brault)

$$
38 \text { (1992), no 4, p. 209-210 }
$$

Répertoire des documents cartographiques et photographiques sur la région de TroisRivières (04), [compilé par] Marie Lefebvre et al. (Yves Tessier)

33 (1987), no 2, p. 74-76

Répertoire des dramatiques québécoises à la télévision, 1952-1977

voir Pagé, Pierre et Legris, Renée

Répertoire des écoles des sciences de l'information

voir Lajeunesse, Marcel

Répertoire des livres de langue française disponibles (1re éd.) 1972- (Jacques Paradis)

19 (1973), no 3, p. 134-136

Répertoire des oeuvres de la littérature radiophonique québécoise, 1930-1970 voir Pagé, Pierre

Répertoire des outils documentaires dans les centres de documentation

voir ASTED. Commission des bibliothèques spécialisées et de recherche

Répertoire des périodiques québécois : 1re partie

voir Bibliothèque nationale du Québec

Répertoire des politiques du prêt entre bibliothèques et des services de reproduction au Canada

voir Services de prêt entre bibliothèques : manuel d'information

Répertoire du livre et de la presse en Afrique

voir The African Book World \& Press : A Directory. Répertoire du livre et de la presse en Afrique. 2nd. ed., rev. and greatly enl.

Le réseau bibliographique informatisé et l'accès au document

voir Chauveinc. Marc

La restauration mineure des documents sur papier; initiation pratique voir Cirkovic-Stanojlovic, Ljiljana

Rétrospective National Bibliographies : An International Directory voir Beaudiquez, Marcelle
Revai, Elisabeth. Alexandre Vattemare trait d'union entre deux mondes. (Marcel Lajeunesse)

21 (1975), no 4, p. $228-229$

Revue d'histoire littéraire du Québec et du Canada. (Denyse Therrien)

$$
33 \text { (1987), no 4, p. } 140-141
$$

Revues d'analyse et d'indexation. Répertoire annoté des revues d'analyse et d'indexation de la collection de la Bibliothèque de l'Université Laval

voir Jolicoeur, Louis-Philippe

Rey, Alain. Dictionnaire des littératures de langue française, par Jean-Pierre de Beaumarchais, Daniel Couty et Alain Rey. (Pierre Giguère)

$$
31 \text { (1985), no 2, p. 74-75 }
$$

(Rheault, François). Houyoux, Philippe. Bibliographie de l'histoire de l'éducation au Québec des origines à 1960, compilée par Philippe Houyoux

25 (1979), no 1, p. 56

(Richer, Suzanne). Pelou, Pierre. L'Europe de l'information

$$
36 \text { (1990), no 3, p. 110-111 }
$$

(Richer, Yvon). Rolland-Thomas, Paule. Règles de catalogage anglo-américaines (...) sous la direction de Paule RollandThomas avec la collaboration de Pierre Deslauriers. Traduction de Rita Bélanger

19 (1973), no 4, p. 177-178

Richter, Noë. Les bibliothèques populaires. (Marcel Lajeunesse)

$$
26 \text { (1980), no 1, p. 56-58 }
$$

Rinfret, Edouard $G$. Le théâtre canadien d'expression française; répertoire analytique des origines à nos jours. (Odette Blouin-Cliche)

$$
22 \text { (1976), no 2, p. } 94
$$

(Rivet-Panaccio, Colette). Bibliothèque nationale du Québec. Répertoire des périodiques québécois : 1re partie, par Ginette Henry

21 (1975), no 4, p. 227-228

Rivet-Panaccio, Colette. Les bibliothèques canadiennes à l'ère de l'automatisation : synthèse bibliographique 1970-72, par Colette Rivet-Panaccio, Amal Awad et Robert Cardinal. (Marcel Fontaine)

19 (1973), no 2, p. 93-94
Roberge, Michel. L'expertise québécoise en gestion des documents administratifs; bibliographie thématique et chronologique 1962-1987. (Jean-Rémi Brault)

34 (1988), no 3, p. 113

(Roberge, Pierre). Vidéoculture 29 (1983), no 2, p. 82-84

Robert, Paul. Dictionnaire alphabétique et analogique de la langue française, $2 e$ éd. (Gilberte Boilard)

32 (1986), no 1-2, p. 53-54

Robin, Xavier. Technologie des systèmes bureautiques. (Philippe Rouyère)

30 (1984), no 4, p. 143-144

Robitaille, Denis. Theses in Canada : A Bibliographic Guide = Thèses au Canada : guide bibliographique, $2 e$ édition, par Denis Robitaille et Joan Waiser. (Claude Fournier)

34 (1988), no 1, p. $37-38$

(Rolland-Thomas, Paule). Classe FC. Cadre de classification de l'histoire du Canada

23 (1977), no 2, p. 112-114

(Rolland-Thomas, Paule). Dewey, Melvil. Abridged Dewey Decimal Classification and Relative Index. Ed. 12, edited by John $P$. Comaroni et al.

37 (1991), no 2, p. $82-83$

(Rolland-Thomas, Paule). Filion, Louise. Classification des publications gouvernementales du Québec. 2e éd.

21 (1975), no 1, p. 58-60

Rolland-Thomas, Paule, éd. Prospectives en bibliothéconomie et en sciences de l'information : mélanges dédiés à la mémoire de Laurent-G. Denis. (Wyley L. Powell)

37 (1991), no 1, p. 41-42

Rolland-Thomas, Paule. Règles de catalogage anglo-américaines (...) sous la direction de Paule Rolland-Thomas avec la collaboration de Pierre Deslauriers. Traduction de Rita Bélanger. (Yvon Richer)

19 (1973), no 4, p. 177-178

Rothwell, Helene de F. A Proposal for a Selective, Annotated Catalogue of Canadian Produced Non-Print Materials. Research Report. (Jacques Demers)

24 (1978), no 4, p. 205-208 
(Rousseau, Denis). Bélanger, Nycole. "La documentation", Méta (numéro spécial), v. 25 , no 1 , (mars 1980), sous la direction de Nycole Bélanger

27 (1981), no 1, p. $37-39$

Rousseau, Françoise Lamy

voir Lamy-Rousseau, Françoise

(Rousseau, Jean-Yves). Gagnon-Arguin, Louise. Une introduction au contrôle d'autorité pour le traitement des noms propres en archivistique

37 (1991), no 3, p. 124-125

Rousseau, Jean-Yves. Les archives au $X X e$ siècle : une réponse aux besoins de l'administration et de la recherche, par Carol Couture et Jean-Yves Rousseau. (Baïla Wane)

30 (1984), no 1, p. 33-35

(Rousseau, Louis-Jean). Colpron, Gilles. Dictionnaire des anglicismes

32 (1986), no 1-2, p. 61-63

(Rousseau, Louis-Jean). Dagenais, Gérard. Dictionnaire des difficultés de la langue française au Canada

32 (1986), no 1-2, p. 61-63

(Rousseau, Louis-Jean). Girodet, Jean. Dictionnaire du bon français 32 (1986), no 1-2, p. 61-63

(Rousseau, Louis-Jean). Hanse, Joseph. Nouveau dictionnaire des difficultés du français moderne

32 (1986), no 1-2, p. 61-63

(Rouyère, Philippe). Robin, Xavier. Technologie des systèmes bureautiques 30 (1984), no 4, p. 143-144

(Rovira, Anna). Catalogue de livres français 1972 / French Book Catalogue 20 (1974), no 3, p. 167-168

(Roy, Jean-Louis). Cain, Julien. Le livre français : hier, aujourd'hui, demain. Ouvrage collectif sous la direction de Julien Cain, Robert Escarpit, Henri-Jean Martin

20 (1974), no 1, p. 44-45

Roy, Jean-Louis. Edouard-Raymond Fabre, libraire et patriote canadien, 17991854. (Jacques Panneton)

21 (1975), no 2, p. 105-106

(Roy, Jean-Luc). Chevalier, Brigitte. Méthodologie d'utilisation d'un centre de documentation

28 (1982), no 1 , p. $39-40$
(Roy, Jean-Paul). Québec. Ministère de l'Éducation. Direction générale de l'évaluation et des ressources didactiques. Direction des ressources didactiques. Les bibliothèques scolaires québécoises. Plus que jamais...

35 (1989), no 4, p. 189-190

$\mathbf{S}$

(Sablonnière, Roger de la). Bernier, Gérald. Le Québec en chiffres de 1850 à nos jours, par Gérald Bernier et Robert Boily

35 (1989), no 3, p. 121-123

(Sablonnière, Roger de la). Fabre, JeanBernard. Le Québec mis en chiffres: statistiques sociales et économiques sur les régions et les villes du Québec

35 (1989), no 3, p. 121-123

(Sablonnière, Roger de la). Langlois, Simon et al. La société québécoise en tendances 1960-1990

38 (1992), no 4, p. 213-214

(Sablonnière, Roger de la). Le Québec statistique, 1985-1986. 58e éd.

32 (1986), no 3, p. 98-99

(Sablonnière, Roger de la). Québec. Bureau de la statistique du Québec. Répertoire de données et de publications statistiques québécoises

30 (1984), no 2, p. $62-63$

(Saint-Germain, Maurice). Brown, Barbara E. rédactrice. Sources d'informations économiques et commerciales canadiennes / Canadian Business and Economics : A Guide to Sources of Information

23 (1977), no 1, p. $47-48$

(Sansfaçon, Jacques). Mailloux, Pierre. Bibliographie annotée d'ouvrages de référence en usage au bureau de la bibliographie rétrospective

20 (1974), no 4, p. 206-207

Sanson, Jacqueline

voir Melet-Sanson, Jacqueline

Santerre, Pierrette. Croc-en-livre rencontre avec les livres. Pour une animation du livre avec les 4-10 ans, par Girnette Tremblay et Pierrette Santerre. (Hélène Charbonneau)

31 (1985), no 4, p. 168-169
(Sauvé,Diane). Nobréga, Gérard. Thésaurus Envirodoq, en collaboration avec Informatech France-Québec

27 (1981), no 3, p. 130-132

(Savard, Pierre). Archives publiques du Canada. Guide des sources d'archives sur le Canada français au Canada

22 (1976), no 3, p. 146-147

(Savard, Réjean). Le Tessier 86 : répertoire 83-86 des documents audiovisuels canadiens de langue française

33 (1987), no 2, p. 73-74

Savard, Réjean. Principes directeurs pour l'enseignement du marketing dans la formation des bibliothécaires, documentalistes et archivistes. (Sylvie Fournier)

35 (1989), no 3, p. 124

Scientific and Technical Societies of Canada / Sociétés scientifiques et techniques du Canada (2e éd.). (Rodolphe Lavergne)

$$
19 \text { (1973), no 1, p. } 42
$$

Seibel, Bernadette. Au nom du livre; analyse sociale d'une profession : les bibliothécaires. (Gilbert Gagnon)

35 (1989), no 2, p. 62-64

Sénécal, André. A Reader's Guide to Québec Studies. (Jean-Rémi Brault)

36 (1990), no 2, p. 77-78

Service coopératif de transmission de l'information sur l'éducation. Liste rotative de descripteurs sur l'éducation. (Claire Renaud-Frigon)

19 (1973), no 1, p. 45-46

Service des bibliothèques publiques voir Québec. Ministère des Affaires culturelles. Service des bibliothèques publiques

Services de prêt entre bibliothèques: manuel d'information. (Johanne Sgroi) 36 (1990), no 4, p. 144-145

Services documentaires multimedia inc. Listes bibliographiques spécialisées correspondantes aux programmes d'études de l'enseignement primaire et secondaire. (Yves Léveillé)

36 (1990), no 2, p. $78-80$

Les services en langue française dans les bibliothèques publiques de l'Ontario voir Desjardins, Louis 
(Sgroi, Johanne). Services de prêt entre bibliothèques: manuel d'information

36 (1990), no 4, p. 144-145

(Simard, Denis). Vers une planification des services québécois d'information documentaire. Textes déposés lors de la Table ronde, 5 mai 1978, présentés par JeanRémi Brault

$$
26 \text { (1980), no 3, p. 169-172 }
$$

La situation des bibliothèques publiques au Saguenay-Lac-Saint-Jean

voir Ouellet, Jacques

Skouri, Yolande. Guide pratique des techniques documentaires, par Claire Guinchat et Yolande Skouri, avec la collaboration de Marie-Pierre Alix. (Richard Paré)

$$
37 \text { (1991), no 2, p. } 83
$$

La Société québécoise en tendances 19601990

voir Langlois, Simon

Sociétés scientifiques et techniques du Canada

voir Scientific and Technical Societies of Canada / Sociétés scientifiques et techniques du Canada

Sociologie du livre et de la lecture. (Gilbert, Gagnon)

25 (1979), no 4, p. 221-225

Soriano, Marc. Guide de la littérature pour la jeunesse. (Alvine, Bélisle)

$$
22 \text { (1976), no 3, p. 150-153 }
$$

(Sorieul, Françoise). ENAP. Centre de documentation. La documentation à caractère international dans les institutions du Québec. Etude préparée par le groupe de travail sur le projet de création au Québec d'un établissement d'études internationales (PROCEI)

30 (1984), no 1, p. $27-28$

(Soucy, François-R.). L'enseignement du français au primaire

$$
27 \text { (1981), no 4, p. 161-164 }
$$

Sources d'information sur la consommation. (Gilles Paré)

$$
23 \text { (1977), no 1, p. } 52-54
$$

Sources d'informations économiques et commerciales canadiennes voir Brown, Barbara E.
St-Amour, Jean-Pierre F. L'Outaouais québécois. Guide de recherche et bibliographie sélective. (Michelle Bélisle-Laflèche)

25 (1979), no 1, p. $51-53$

Stanojlovic, Ljiljana Cirkovic

voir Cirkovic-Stanojlovic, Ljiljana

Statistiques culturelles du Québec 19711982. (Gilbert Gagnon) 32 (1986), no 3, p. 100-101

Statistiques de l'édition au Québec en 1990 voir Québec. Bibliothèque nationale du Québec

Structures, organisation et animation culturelle dans les bibliothèques en Côte d'Ivoire

voir Amoikon, Michel K.

Such, Marie-France. Initiation à la bibliographie scientifique, par Marie-France Such et Dominique Perol. (Claude Busque) 35 (1989), no 1, p. 28-29

Les Sulpiciens et la vie culturelle à Montréal au XIXe siècle voir Lajeunesse, Marcel

Le système ESAR

voir Garon, Denise

(Szpakowska, Janina-Klara). Allard, Yvon. Paralittérature 1

22 (1976), no 1, p. $45-47$

Szpakowska, Janina-Klara. Besoins en information et en services documentaires chez les jeunes de 14-19 ans. (Christiane Charette)

$$
26 \text { (1980), no 1, p. 53-55 }
$$

Szpakowska, Janina-Klara. Ces lectures sauvages; panoramique sur le service de bibliothèque aux jeunes adultes. (Marielle Durand)

19 (1973), no 2, p. 89-91

Szpakowska, Janina-Klara. Jeunes gens : thésaurus; essai de systématisation des connaissances spécifiques à la classe d'âge 13-25 ans. (Rénald Buteau)

35 (1989), no 3, p. 123-124

Szpakowska, Janina-Klara. Profil d'un centre documentaire multimedia. (Georgette Lapointe)

22 (1976), no 2, p. 95-97
$T$

Tacchini, Catherine. La lecture publique en Suisse romande : enquête du groupe de travail des bibliothèques de lecture publique, par Béatrice Laserre et Catherine Tacchini. (Réal Messier)

25 (1979), no 4, p. 225-227

Tamaro, Normand. La loi sur le droit d'auteur commentée et annotée. (Hugues Létourneau)

38 (1992), no 3, p. 171

Tardif, Evelyne. Guide de recherche documentaire en science politique, par Mohamed Laghzali et Evelyne Tardif. (Gaston Bernier)

34 (1988), no 2, p. $80-81$

Technologie des systèmes bureautiques voir Robin, Xavier

Tega, Vasile. Information et documentation, facteurs de progrès dans l'entreprise. (Philippe Houyoux)

22 (1976), no 2, p. 97-98

La télématique documentaire à l'épreuve. Usages et usager des bases de données à la BPI

voir Coulaud, Philippe

Tesauro O.I.T.

voir Bureau international du travail

Le Tessier 86 : répertoire $83-86$ des documents audiovisuels canadiens de langue française. (Réjean Savard)

33 (1987), no 2, p. 73-74

(Tessier, Yves). Bellemare, Louis. L'information électronique au Québec : guide pratique des services d'information en ligne, par Louis Bellemare et Léo Filion

33 (1987), no 4, p. 137-138

(Tessier, Yves). Carto-03 : Répertoire cartobibliographique sur la région de Québec, compilé par Yves Tessier avec la collaboration de Jacques Martinez et la participation de Louise Lavoie

33 (1987), no 2, p. 74-76

(Tessier, Yves). Cossette, Claude. La publicité en action : comment élaborer une campagne de publicité ou ce qui se passe derrière les murs d'une agence, par Claude Cossette et René Déry

34 (1988), no 1, p. $39-40$ 
(Tessier, Yves). Documents cartographiques depuis la découverte de l'Amérique jusqu'à 1820 : inventaire sommaire, [compilé par] Pierre Lépine et Josée Berthelette

33 (1987), no 2, p. 74-76

(Tessier, Yves). Infor-Carto 02 : Répertoire cartobibliographique sur la région 02 et Moyen Nord, par Guy Biron et France Gagnon sous la direction de Richard Bouchard

33 (1987), no 2, p. 74-76

(Tessier, Yves). Répertoire cartobibliographique de Charlevoix, [compilé par] Daniel Dufour

33 (1987), no 2, p. 74-76

(Tessier, Yves). Répertoire des documents cartographiques et photographiques sur la région de Trois-Rivières (04), [compilé par] Marie Lefebvre et al.

33 (1987), no 2, p. 74-76

Tessier, Yves, comp. Répertoire des atlas de la cartothèque, compilé par Yves Tessier. (Pierre Lépine)

20 (1974), no 2, p. 106-107

Le Tessier; répertoire des documents audiovisuels canadiens de langue française. (Jacques Paradis)

30 (1984), no 1, p. $30-31$

Tétreault, Raymond. Le livre dans la vie de l'enfant. Actes du Colloque 1977. Textes colligés et présentés par Raymond Tétreault. (France Latreille-Huvelin)

27 (1981), no 1, p. $36-37$

Le théâtre canadien d'expression française; répertoire analytique des origines à nos jours

voir Rinfret, Edouard G.

Le Théâtre canadien-français; évolution, témoignages, bibliographie

voir Université d'Ottawa. Centre de recherche en civilisation canadiennefrançaise

Théorie et pratique dans l'enseignement des sciences de l'information. Bridging the Gap between Theory and Pratice. Textes colligés par Réjean Savard. (Michèle Hudon)

35 (1989), no 2, p. 61-62

(Thériault, Jacqueline). Choix Jeunesse. Jeux et Jouets 1981

29 (1983), no 3, p. 122-124
(Thériault, Jacqueline). Choix Jeunesse. Jeux et Jouets 1982

29 (1983), no 3, p. 122-124

Thériault, Michel. Choix et acquisition des documents au Québec. Volume 1 : Principes et applications. (Gilles Bergevin)

23 (1977), no 3, p. 169-170

Thériault, Michel. Le livre religieux au Québec depuis les débuts de l'imprimerie jusqu'à la Confédération (1764-1867) : relevé statistique et essai d'interprétation. (Gilles Gallichan)

24 (1978), no 1, p. $48-49$

Thériault, Michel. Les instituts de vie consacrée au Canada depuis les débuts de la Nouvelle-France jusqu'à aujourd'hui; notes historiques et références. The Institutes of Consacred Life in Canada from the Beginning of the New France up to the Present; Historical Notes and References. (Jean-Rémi Brault)

27 (1981), no 3, p. 132

Thériault, Yvon. Les publications parlementaires d'hier et d'aujourd'hui. 2 e éd. (Gilbert Lefebvre)

30 (1984), no 2, p. 61

(Therrien, Denyse). Revue d'histoire littéraire du Québec et du Canada 33 (1987), no 4, p. 140-141

Thésaurus : Enfance inadaptée

voir Dubuc, Pierrette

Thésaurus B.I.T

voir Bureau international du travail

Thésaurus Envirodoq

voir Nobréga, Gérard

Thésaurus. délinquance juvénile voir Blazina, Vesna

Thèses au Canada : guide bibliographique, $2 e$ édition

voir Robitaille, Denis

Theses in Canada: A Bibliographic Guide = Thèses au Canada : guide bibliographique, $2 e$ édition

voir Robitaille, Denis

Thibault, Danielle. Guide de rédaction bibliographique. (Réal Bosa)

37 (1991), no 3, p. 122
(Thibault, Jean). Buchinski, Edwin J. Le contexte de linterconnexion dans le cadre de l'élaboration d'un réseau bibliographique national

27 (1981), no 3, p. 128

Thibodeau-Brunet, Lise. Thésaurus : Enfance inadaptée, par Pierrette Dubuc, Lise Thibodeau-Brunet et Luc-Louis Lecompte. (Jean Bouthillette et Raymond Caron)

27 (1981), no 2, p. 82-83

Thomas, Paule Rolland

voir Rolland-Thomas, Paule

Traitement automatisé des documents multimedia avec les systèmes ISBD unifié, Lamy-Rousseau et PRECIS. Proposition SILP

voir Lamy-Rousseau, Françoise

Le traitement linguistique de l'information documentaire: l'analyse documentaire voir Chaumier, Jacques

Travail et méthodes du / de la documentaliste

voir Chaumier, Jacques

Travaux du 10e congrès tenu à Montréal du 27 au 30 octobre 1983. Au-delà de l'information : la communication

voir Association pour l'avancement des sciences et des techniques de la documentation

(Tremblay, Clément). Clavel, Jean-Pierre et al. L'évaluation des bibliothèques universitaires

32 (1986), no 1-2, p. 58-59

Tremblay, Ginette. Croc-en-livre : rencontre avec les livres. Pour une animation du livre avec les 4-10 ans, par Ginette Tremblay et Pierrette Santerre (Hélène Charbonneau)

31 (1985), no 4, p. 168-169

(Turgeon, Jean-Marc). Dewey, Melvil. Classification décimale de Dewey et index, établis par Melvil Dewey

21 (1975), no 1, p. 60-62

U

Un titre s'il vous plaît : conseils pratiques à l'intention des éditeurs de revues spécialisées, rapports, journaux et autres publications en série canadiennes voir Franklin, Karen 
Une agence de presse au Québec? Nécessité, utilité et viabilité économique. (Rapport)

voir Multi-Réso Inc.

Une bibliothèque pour Montréal; rapport préléminaire sui la possibilité d'établir une bibliothèque publique à la Place Desjardins voir Castonguay, André

Une introduction au contrôle d'autorité pour le traitement des noms propres en archivistique

voir Gagnon-Arguin, Louise

Une invitation : la bibliothèque scolaire mise à profit. Réalisation, Anne Bernier. (Paulette Bernhard et Patrick Delobel)

34 (1988), no 3 , p. $109-110$

Une sélection de 100 films tirés de romans en vidéocassettes

voir Bérubé, Robert-Claude

Une societé sans papier

voir Gallouédec-Génuys, Françoise

Uniformisation des règles de catalogage des documents visuels et sonores description d'une expérience

voir Lamy-Rousseau, Françoise

Union des écrivains québécois. Petit dictionnaire des écrivains. (Michèle Hudon) 26 (1980), no 1, p. 55-56

Union List of Manuscripts in Canadian Repositories

voir Archives publiques du Canada

Union List of Serials in the Montreal Area $=$ Catalogue collectif des périodiques de la région de Montréal, 1986. (Nicole Lavigne) 32 (1986), no 1-2, p. $57-58$

Université d'Ottawa. Centre de recherche en civilisation canadienne-française. Le théâtre canadien-français; évolution, témoignages, bibliographie. (Monique Desrochers)

24 (1978), no 3, p. 143-145

Université Laval. Bibliothèque. Service de catalogage. Index anglais-français des termes utilisés dans le Répertoire de vedettes-matière. (Georges-A. Chartrand)

19 (1973), no 4, p. 178-181

Université Laval. Bibliothèque. Service de catalogage. Répertoire de vedettes-matière (7e éd.). (Georges-A. Chartrand)

19 (1973), no 4, p. 178-181
L'utilisation des moyens didactiques imprimés et audiovisuels par les professeurs au secondaire : une étude préliminaire

voir Bélanger, Marcel

V

Vachet, André. Les idéologies au Québec, par Denis Monière et André Vachet. (Daniel Rémi)

23 (1977), no 1, p. $51-52$

(Vadnais, Ginette). Gauvin, Daniel. Guide canadien du livre rare / Canadian Guide to Rare Books

$$
36 \text { (1990), no 3, p. } 109-110
$$

(Vadnais, Ginette). Lassonde, Jean-René. La bibliothèque Saint-Sulpice, 1910-1931 33 (1987), no 2, p. 72-73

Van der Bellen, Liana)

$$
27 \text { (1981), no 1, p. } 35-36
$$

Van Slype, Georges. Conception et gestion des systèmes documentaires. (Laval Du Breuil)

$$
24 \text { (1978), no 4, p. 208-209 }
$$

(Vermette, Jacques). Dubuc, Robert. Vocabulaire bilingue de la production télévision

$$
29 \text { (1983), no 3, p. } 124
$$

(Véronneau, Pierre). Houle, Michel. Dictionnaire du cinéma québécois, par Michel Houle et Alain Julien

25 (1979), no 3, p. 165-167

Véronneau, Pierre. Écrits sur le cinéma : bibliographie québécoise 1911-1981, par Madeleine Fournier-Renaud et Pierre Véronneau. (Régis Mathieu)

$$
30 \text { (1984), no 3, p. 103-104 }
$$

Véronneau, Pierre. Les cinémas canadiens. Dossier établi sous la direction de Pierre Véronneau. (Julien, Alain)

25 (1979), no 3, p. 164-165

Vers l'établissement d'un réseau de communication bibliographique au Canada voir Durance, Cynthia J.

Vers un réseau québécois des bibliothèques. Rapport du CERBIQ

voir Québec. Comité d'étude d'un réseau des bibliothèques québécoises pour le traitement en coopération de leur documentation
Vers une planification des services québécois d'information documentaire. Textes déposés lors de la Table ronde, 5 mai 1978

voir Brault, Jean-Rémi

Veyron, Michel. Dictionnaire canadien des noms propres. (Gaston Bernier)

37 (1991), no 4, p. 164-165

Viatte, A. Dictionnaire général de la francophonie, par J.-J. Luthi, A. Viatte, G. Zanarini. (Gilles Gallichan)

34 (1988), no 2, p. $79-80$

Vidéoculture (Pierre Roberge)

29 (1983), no 2, p. 82-84

La vie littéraire au Québec

voir Lemire, Maurice

Viens de paraître. v. 1- 1965(Jacques Paradis)

19 (1973), no 3, p. 134-136

Ville de Montréal. Service des activités culturelles. Division des bibliothèques. Catalogue des films. (Louise de Chevigny) 32 (1986), no 4, p. 157-158

Ville de Montréal. Service des activités culturelles. Division des bibliothèques. Catalogue des vidéocassettes. (Louise de Chevigny)

32 (1986), no 4, p. 157-158

Vincke, Christian. Problèmes de droit d'auteur en éducation, par Christian Vincke. Avec la collaboration de Pierre A. Côté et Victor Nabhan. (Claudio Antonelli)

25 (1979), no 1, p. 49-50

Vinet, Bernard. Bibliographie sur les personnes âgées : répertoire des livres et des périodiques de la collection de la Bibliothèque de I'Université Laval, par Bernard Vinet et Louis-Philippe Jolicoeur. (Pierrette Galarneau)

24 (1978), no 3, p. 151-152

Vlach, Milada. Catalogue collectif des impressions québécoises. 1764-1820, par Milada Vlach et Yolande Buono. (Gilles Gallichan)

30 (1984), no 4, p. 142-143

Vlach, Milada. Laurentiana parus avant 1821, par Milada Vlach, avec la collaboration de Yolande Buono. (John E. Hare)

23 (1977), no 4, p. 215-216 
Vocabulaire bilingue de la production télévision

voir Dubuc, Robert

Vocabulaire libre en indexation voir Blais, Raymond

\section{w}

Waiser, Joan. Theses in Canada : A Bibliographic Guide = Theses au Canada : guide bibliographique, 2e édition, par Denis Robitaille et Joan Waiser. (Claude Fournier)

34 (1988), no 1, p. $37-38$

(Waller, Marc). Bonville, Jean de. Bibliocom 1976; bibliographie internationale de la documentation en langue française sur la communication 26 (1980), no 2, p. 117-118

(Waller, Marc). Bonville, Jean de. Bibliocom 1977; bibliographie internationale de la documentation en langue française sur la communication

26 (1980), no 2, p. 117-118

Walne, Peter et al. Dictionary of Archival Terminology = Dictionnaire de terminologie archivistique. English and French with Equivalents in Dutch, German, Italian, Russian and Spanish. (Louise GagnonArguin et Carol Couture)

32 (1986), no 3, p. 101-102
(Wane, Baïla). Couture, Carol. Les archives au $\mathrm{XXe}$ siècle : une réponse auX besoins de l'administration et de la recherche, par Carol Couture et Jean-Yves Rousseau

30 (1984), no 1 , p. $33-35$

(Ward, W. Dale). The African Book World \& Press : A Directory. Répertoire du livre et de la presse en Afrique. 2 nd. ed., rev. and greatly enl.

27 (1981), no 4, p. 158-159

Warwick, Peat. La fréquentation des bibliothèques et des librairies au Canada, par Michel Ben-Gera, Peat Warwick and Partners et Brian L. Kingsley. (Gilbert Gagnon)

27 (1981), no 4, p. 164-166

Wenger, Isabelle. Bibliothèques dans la rue. Une expérience des bibliothèques municipales de la Ville de Genève, par Catherine Barut et Isabelle Wenger. (Christiane Charette)

33 (1987), no 2, p. 71-72

(Wilson, Lise). Québec. Assemblée nationale. Bibliothèque de la Législature. Le référendum : bibliographie sélective et annotée

24 (1978), no 1, p. $43-44$

Woodrough, Lise Calvé voir Calvé-Woodrough, Lise
Wyczynski, Paul. Dictionnaire pratique des auteurs québécois, par Réginald Hamel, John Hare et Paul Wyczynski. (Patrick Coppens)

23 (1977), no 2, p. 109-112

\section{Z}

Zanarini, G. Dictionnaire général de la francophonie, par J.-J. Luthi, A. Viatte, G. Zanarini. (Gilles Gallichan)

34 (1988), no 2, p. $79-80$

Zell, Hans M. African Books in Print. Livres africains disponibles. (Théo Nkembé)

26 (1980), no 4, p. 224-225

Zundel-Ben-Khemis, Anne. Ouvrages de référence pour les bibliothèques publiques : répertoire bibliographique, par Marielle Beaudiquez et Anne Zundel-Ben-Khemis. (Gaston Blais)

21 (1975), no 2, p. 108-109 